\title{
Fazit: Die Entstehung von Themen in der Planungswissenschaft
}

In diesem Kapitel werden die Ergebnisse der Arbeit zusammengetragen und die Forschungsfrage , Wie entstehen Themen in der Planungswissenschaft?' in komprimierter Form beantwortet. Dafür werden zunächst die empirischen Befunde zu den Themenkarrieren Schrumpfende Städte und Klimawandel in eine allgemeine Beschreibung der Entstehung von Themen in der Planungswissenschaft überführt. Die eigentliche Funktion der Phasenheuristik kommt hier zum Tragen: Durch sie werden die empirischen Analysen zu den beiden Themenkarrieren in jeweils vergleichbare Segmente aufgeteilt, sodass die konkreten Ereignisse und Prozesse in einem synoptischen Phasenverlauf eingeordnet werden können (siehe Kp. 9.1). Auf dieser Grundlage wird nach den Faktoren geforscht, die maßgeblichen Einfluss auf die Entwicklung der fachgemeinschaftlichen Aufmerksamkeit gegenüber den beiden Themenverläufen hatten. Hier werden generalisierbare Mechanismen abgeleitet, die den beiden planungswissenschaftlichen Themenkarrieren zu Grunde liegen (siehe Kp. 9.2). Schließlich wird auf die Auswirkungen eingegangen, die die temporären Fokussierungen auf die planungswissenschaftliche Disziplin haben (siehe Kp. 9.3).

\subsection{Die Entstehung von Themen in der Planungswissenschaft: Von der Nichtbeachtung zum unhinterfragten Kontext}

Im Folgenden wird zur Beantwortung der Forschungsfrage der Verlauf einer planungswissenschaftlichen Themenkarriere dargelegt. Diese Schilderung stellt einerseits die Synopse der beiden untersuchten Themenkarrieren Schrumpfende

A. Gravert, Themenkarrieren in der Wissenschaft, 
Städte und Klimawandel dar. Andererseits legt sie hypothesenartig eine idealtypische Abfolge von Mechanismen offen, so dass Aufmerksamkeitsverläufe gegenüber anderen Themen auf dieser Basis untersucht und verglichen werden können.

Um Missverständnissen entgegenzuwirken, ist - gerade angesichts der starken Ähnlichkeit der beiden erhobenen Fallbeispiele (siehe Abb. 9.1) - auf Einschränkungen in Bezug auf die Allgemeingültigkeit der vorgelegten Erkenntnisse hinzuweisen. So nimmt nicht jedes Thema in der Planungswissenschaft den geschilderten linearen Verlauf. Es ist davon auszugehen, dass einige Themen nie disziplinweite Aufmerksamkeit erlangen, dass einem Durchbruch und einer zwischenzeitlichen Fokussierung keine Phase der weitläufigen Ignoranz unmittelbar vorausgeht und dass andere Ausprägungen der Fokusphase bezüglich Dauer und Dimension beobachtet werden können. Ungeachtet dessen werden Mechanismen der wissenschaftlichen Aufmerksamkeitsentstehung dargelegt, die auch unabhängig von Phasenabfolge, Intensität oder Dimension analytisch auf andere Themen angewendet werden können. Darüber hinaus dient diese Arbeit dem Nachweis, dass Themenzyklen in eben jener geschilderten, in gewisser Hinsicht extremen, Form auftreten. Hierfür ein Bewusstsein zu schaffen und eine Diskussion darüber anzustoßen (siehe insb. Kp. 10) ist ein Ziel dieser Arbeit.

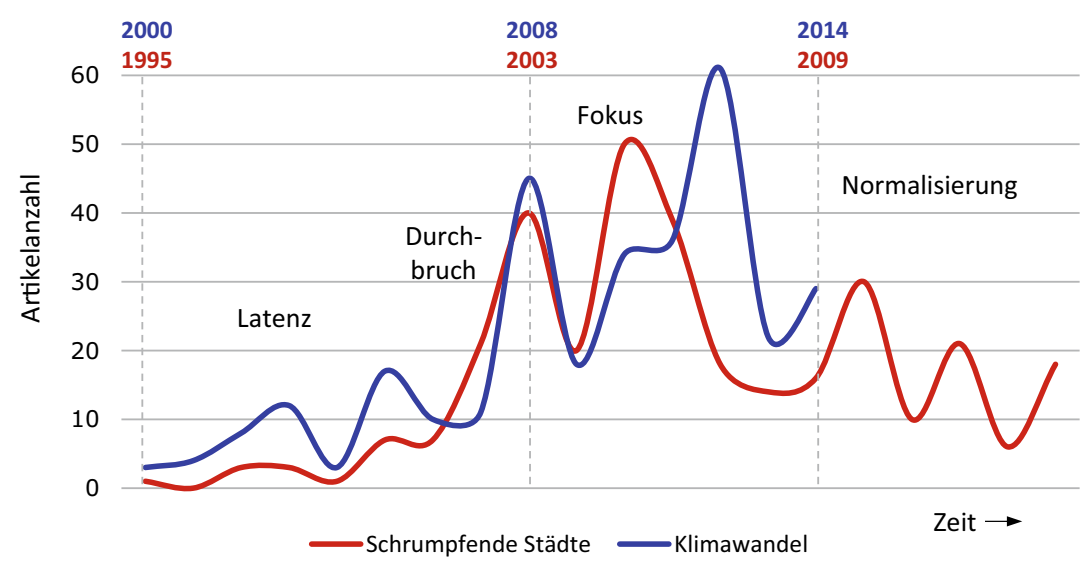

Abb.9.1 Aufmerksamkeitskurven der beiden Themen Schrumpfende Städte und Klimawandel übereinandergelegt (Quelle: Eigene Erhebung auf Basis der planungswissenschaftlichen Zeitschriften 1995-2014) 
Zudem sind beide untersuchten Themenkarrieren das Resultat multidimensionaler Prozesse. Sie werden im Folgenden in ihrer Komplexität (weiter) reduziert, mit dem Ziel generalisierbare Faktoren herauszustellen. Zu der Multidimensionalität gehört, dass ein Thema Teil anderer Themen ist, genauso wie es verschiedene Subthemen unter sich vereint. So setzt sich bspw. die Themenkarriere Klimawandel aus verschiedenen, interagierenden Themenkarrieren mit ganz unterschiedlichen Verläufen zusammen, darunter Nachhaltigkeit, Elbe-Hochwasser, Energiewende, Umweltprüfung, adaptive Kapazität, Resilienz. und viele mehr. Jedes dieser Themen bringt nicht nur eigene gesellschaftliche Impulse, eigene Akteur*innen und einen eigenen Aufmerksamkeitsverlauf in den Klimawandeldiskurs ein, sondern auch spezifische inhaltliche Verschiebungen, so dass die Schnittmengen zwischen den Themen unterschiedlich stark ausgeprägt sind. Auch gehört zu jeder Themenkarriere eine spezifische räumliche Komponente. So war bspw. das Thema Schrumpfung zunächst auf Ostdeutschland beschränkt und hat durch die spätere Diskurserweiterung auf Westdeutschland einen starken Impuls bekommen. Diese Spezifika gehen durch die Abstraktion zwar verloren, dafür wird aber der Blick für themenübergreifend vergleichbare Mechanismen freigemacht.

Die Schilderung der Themenkarrieren erfolgt in der empirisch bewährten Phaseneinteilung - Latenzphase (Kp. 9.1.1), Durchbruchphase (Kp. 9.1.2), Fokusphase (Kp. 9.1.3) und Normalisierungsphase (Kp. 9.1.4) (siehe Abb. 9.1). Um die Klarheit der Schilderung zu gewährleisten, wird innerhalb jeder Phase eine Gliederung in (1.) Institutioneller Kontext, (2.) Akteur*innen, (3.) Inhalt und (4.) Fazit und Phasenübergang vorgenommen. Sie entspricht dem im Akteurzentrierten Institutionalismus eingehend beschriebenen Spannungsverhältnis zwischen Struktur und Akteur*innen (siehe Kp. 5.2) und ergänzt dieses um den Ausgangspunkt und das Produkt des sozialen Systems der Wissenschaft: die Entwicklung wissenschaftlichen Wissens. Allerdings sind die innerhalb der Phasen geschilderten Prozesse eng miteinander verwoben, weshalb die Grenzen zwischen den Teilkapiteln als fließend zu betrachten sind. Auch soll kein einseitiger Kausalmechanismus suggeriert werden - etwa: Der institutionelle Wandel löst eine Veränderung der Akteur*innenkonstellationen aus, was zu einer inhaltlichen Verschiebung führt. Schließlich lösen auch inhaltliche Verschiebungen einen institutionellen Wandel aus, genauso wie die jeweils getrennt voneinander dargelegten Mechanismen parallel und miteinander verschränkt ablaufen, ohne dass eine eindeutige kausale Abfolge identifiziert werden kann. 


\subsubsection{Latenzphase}

In der Latenzphase beginnen sich verschiedene neue Problemstellungen, die für die Planungswissenschaft grundlegende Bedeutung erlangen könnten, herauszuschälen - bspw. im Rahmen von Bevölkerungsprognosen, naturwissenschaftlichen Erkenntnissen oder exemplarischen Einzelereignissen. Sie werden in der Planungswissenschaft bereits aufgegriffen und in das Bezugssystem der Raumplanung übersetzt. Allerdings sind die Vorstöße selten und erhalten geringe Resonanz innerhalb der Planungswissenschaft sowie in der Planungspraxis. Die Zusammenführung und Verdichtung zu dem Sinnkomplex, der später eine Themenkarriere erfährt, erfolgt erst in der Durchbruchphase sowie unmittelbar davor. Ist also im Folgenden von einem Thema die Rede, so handelt es sich um die ex-post-Betrachtung eines Netzwerks von Subthemen, dessen Verknüpfung und Verdichtung noch aussteht.

\section{Institutioneller Kontext}

Später zusammengehörende Problemstellungen werden in Politik, Öffentlichkeit und Wissenschaft primär in Verbindung mit Einzelereignissen thematisiert. Weder die enge Verknüpfung der einzelnen Aspekte zu einem Thema noch die Dimension der Aufmerksamkeit, die dieses in den verschiedenen gesellschaftlichen Teilsystemen erregen wird, sind bislang absehbar.

Strukturell wird die Beschäftigung mit dem Thema im Rahmen der planungswissenschaftlichen Erkenntnisproduktion sanktioniert. In Öffentlichkeit, Politik und Praxis bleibt die Aufmerksamkeit gegenüber dem Thema gering. Exogene Impulse auf das Anreizsystem der Wissenschaftler*innen geben anderen Themen Vorrang, die als opportuner, dringlicher oder zukunftsträchtiger gelten: In Auftragsforschungen, Gutachten, Plänen, Gesetzgebungen und Medienberichten werden andere Prioritäten gesetzt. Unter den Akteur*innen, die über die thematische Ausrichtung raumplanungsrelevanter Förderprogramme in Wissenschaft und Praxis bestimmen, hat das Thema keine starken Fürsprecher*innen und wird, sofern es überhaupt auf die Entscheidungsagenda gelangt, zugunsten anderer Themen zurückgestellt.

Auch innerhalb der Planungswissenschaft fällt die Ressourcenallokation bspw. in der Ausrichtung von Konferenzen, der Setzung von institutsinternen Forschungsschwerpunkten, der Lehrplanentwicklung oder der Ausgestaltung von Berufungsverfahren - in der Latenzphase grundsätzlich zugunsten bereits etablierter Themen aus. Aufgrund der Neuartigkeit des Themas fehlt das Publikum, das heißt die themenbezogene Fachgemeinschaft, die einschlägige Beiträge rezipieren, bewerten und durch Zitierung oder positive Begutachtung belohnen 
könnte. Häufig erleben die Pionier*innen als Reaktionen auf ihre Kommunikationen Desinteresse, Ridikülisierung und Ignoranz seitens etablierter Akteur*innen der Planungswissenschaft, -politik und -praxis. Eine weitere, anders gelagerte Form der Ablehnung des neuen Themas ist die Tabuisierung. Hierbei erkennen Akteur*innen das Problem bereits in Ansätzen, allerdings lehnen sie es ab, die Bedeutung des Themas explizit einzugestehen. Teilweise sabotieren etablierte Wissenschaftler*innen sogar einschlägige Forschungen im Rahmen ihrer Verfügungsgewalt. Ihre wissenschaftliche Autorität ist in gewissem Maße an die Gültigkeit des vorherrschenden Paradigmas gebunden, das sie nun im Kontext ihres Forschungsfeldes durch das aufkommende Thema in Frage gestellt sehen. Sie versuchen ihre Deutungshoheit, ihre Wissensansprüche und ihr Ansehen zu bewahren. Auch die emotionale Verbundenheit mit dem eigenen Lebenswerk spielt dabei eine Rolle. Zudem herrschen ähnliche Pfadabhängigkeiten in Politik und Praxis vor, weshalb hier auch Abhängigkeiten, etwa in der Auftrags- und Mittelvergabe, ausschlaggebend für die Aufrechterhaltung des Status Quo sein können.

Vor dem Hintergrund dieser strukturellen Hemmnisse ist nachvollziehbar, dass die Akteur*innen ihre Fachbeiträge im Kontext bereits etablierter planungswissenschaftlicher Diskurse rahmen. Sie finden so mit der eigenen Forschung eher Akzeptanz bei bestimmten Gatekeeper*innen, wie bspw. Verlagen, Fördermittelgeber*innen, Gutachter*innen oder Berufungskommissionen. Sie können darüber hinaus das neue Thema an den etablierten Wissenskanon der Planungswissenschaft andocken und die eigene Forschung in einer Fachgemeinschaft verankern, die dann wiederum die eigenen Erkenntnisse rezipiert. Nicht zuletzt geht die Verortung in etablierten Wissensstrukturen auf die eigene Forschungsbiografie zurück, die wiederum aus diesen disziplinären Wissensstrukturen hervorgeht. Hat sich ein*e Wissenschaftler*in also bislang vorrangig mit einem etablierten Forschungspfad befasst, so ist es für ihn*sie nicht nur strategisch sinnvoll an diesen Forschungspfad anzuknüpfen. Die Option, die Forschung in einem neuen, bislang kaum erkennbaren Diskurs zu rahmen, wird gar nicht unbedingt erwogen. Folglich geben etablierte Themenbestände im Sinne eines Paradigmas einen Rahmen für die Erkenntnisproduktion der Fachgemeinschaft vor, der durch die daraus resultierenden Forschungsentscheidungen ihrer Mitglieder kontinuierlich reproduziert wird.

\section{Akteur*innen}

Die Diffusion des Themas in der Planungswissenschaft erfolgt in der Latenzphase durch direkten Kontakt. Häufig geht diesem Kontakt das Entstehen einer persönlichen Faszination für das Thema voraus, dieses kann aber bereits Jahre 
zurückliegen und nahezu folgenlos für die eigene wissenschaftliche Aktivität bleiben. Für das planmäßige Aufgreifen im Kontext der wissenschaftlichen Wissensproduktion sind dagegen direkte Austauschbeziehungen im Kontext der Wissenschaft ausschlaggebend, da sie unmittelbar die Relevanz des Themas für die wissenschaftliche Erkenntnisproduktion erkennbar machen. Es entwickeln sich Kaskaden von direkten fachbezogenen Transaktionen, die zumeist auf Impulse von außerhalb der deutschsprachigen planungswissenschaftlichen Fachgemeinschaft zurückgehen - bspw. aus der Wirtschaft, Politik, anderen Disziplinen oder auf ausländische Fachkolleg*innen. Auch eine Rolle spielen informelle und formelle Austauschformate innerhalb von Forschungseinrichtungen. Auf diese Art entstehen mehrere unverbundene, einschlägig interessierte Teilgruppen - bspw. Lehrstuhlinhaber*in-Mitarbeiter*in, Dozent*in-Student*in oder Arbeitskreise mit thematischen Schnittmengen zu dem entstehenden Diskurs.

Die Pionier*innen sind fasziniert von dem Thema, was unter anderem auf die Diskrepanz zwischen den eigenen Erkenntnissen und der fachgemeinschaftlichen Nichtbeachtung zurückzuführen ist. Die strukturellen Hemmnisse wirken einerseits demotivierend und lenken die Aufmerksamkeit auf andere Themen, deren Bearbeitung einfacher und gewinnbringender erscheint. Auch geben sie einigen Pionier*innen immer wieder Anlass, die Relevanz des eigenen Themas in Frage zu stellen. Andererseits hat gerade die strukturelle Ignoranz einen motivierenden Effekt: Je mehr Ablehnung sie erhalten, desto überzeugendere Argumentationsgrundlagen für die Verhandlung des Themas müssen sie aufbauen. Da sie zunehmend von der Wichtigkeit des Themas überzeugt sind, investieren sie Zeit und Ressourcen, um die benötigten Kompetenzen zu erlangen. Angesichts der ohnehin geringen Resonanz haben die Pionier*innen dabei allerdings keinen Zeitbzw. Handlungsdruck und befassen sich schwerpunktmäßig mit anderen Forschungspfaden, bei denen die Anerkennung eigener Forschungsbeiträge durch das persönliche Umfeld, die Fachgemeinschaft und den*die Geldgeber*in sicherer erscheint.

Einige der Akteur*innen und Teilgruppen wenden sich nach wenigen Jahren einschlägiger Forschung wieder anderen Themen zu. Bei Ihnen stellt sich das Gefühl ein, die wichtigen Fragen in Bezug auf das Thema seien beantwortet. Diese epistemische Erklärung für das Aufgeben der einschlägigen Forschungsaktivität ist unübersehbar an das Ausbleiben von Resonanz geknüpft. So legen die Akteur*innen das Thema ausschließlich in der Latenz- und der Normalisierungsphase ab, also immer nur dann, wenn einschlägige Forschungen im Vergleich mit anderen Themen eine strukturelle Vernachlässigung erfahren. Insbesondere in der Latenzphase werden thematische Vorstöße kaum seitens der Fachgemeinschaft aufgegriffen und geprüft, weitergeführt oder auch in Frage gestellt. Den 
Pionier*innen wird also wenig Inspiration und Motivation zur Vertiefung des Themas vermittelt. Hinzu kommt die geringe Aussicht auf soziales, ökonomisches und politisches Kapital.

Kognitive Bezüge und soziale Beziehungen zwischen den einzelnen Teilgruppen sind in der Latenzphase nicht besonders häufig. Allerdings beginnen sie im Vorfeld der Durchbruchphase ihre einschlägigen Kommunikationen gegenseitig wahrzunehmen. Hierin sind - im Nachhinein - die Anfänge der emergierenden, themenbezogenen Fachgemeinschaft zu erkennen. Eine kritische Masse an Interessierten, die sich durch gegenseitiges Zitieren Reputationsgewinne verschaffen könnte oder die im Sinne einer Epistemischen Gemeinschaft gemeinsam innerhalb und außerhalb der Wissenschaft Überzeugungsarbeit leisten könnte, ist damit aber noch nicht erreicht. Dies kann sich durch die Einberufung regionsübergreifender Arbeitskreise ändern. Sie dienen nicht nur als Forum für die multiperspektivische, systematische Erschließung des neuen Themas. Sie sind auch ein Instrument zur Verknüpfung bislang kaum verbundener Bereiche der einschlägigen Wissensproduktion. Zudem können interregionale Arbeitskreise einen inhaltlichen Bezugsrahmen sowie einen sichtbaren Expert*innenkreis etablieren, sodass das Thema für Akteur*innen innerhalb und außerhalb der Planungswissenschaft personell und inhaltlich greifbar wird.

\section{Inhalt}

Die Verknüpfung mehrerer Teilaspekte zu dem dichten Sinnkomplex, der später eine Themenkarriere erlebt, ist bislang wenn überhaupt nur in Ansätzen erkennbar. Zum einen werden ohnehin nur selten einschlägige Fachbeiträge veröffentlicht. Zum anderen kommt für die Zusammenführung von Erkenntnissen zu einem kohärenten Diskurs erschwerend hinzu, dass die einschlägigen Fachbeiträge zunächst in anderen Diskursen gerahmt werden: Die unterschiedlichen Facetten des Themas werden als Teilaspekt und als Begründungszusammenhang zur Herstellung der Relevanz anderer, bereits fachöffentlich etablierter Themen angeführt. Durch die Rahmung im Kontext verschiedener Fachdiskurse können die einzelnen Fachbeiträge kaum als zusammengehörig identifiziert werden.

Vereinzelt werden die Zusammenhänge zwischen dem Thema und der Raumplanung im Rahmen weniger reputationsträchtiger Formate - bspw. Vorträge, Jubiläumsbände oder planungspraktisch orientierte Zeitschriften - beleuchtet. Mehrjährige, planmäßige und methodisch kontrollierte Auseinandersetzungen mit dem Thema finden seltener statt, können sich aber als besonders grundlegend im einschlägigen Diskurs der Folgejahre erweisen, indem sie bereits die systematische Evaluation bestehender Wissensbestände, Forschungslücken und möglicher Diskussionslinien leisten sowie die Herstellung kognitiver Bezüge und sozialer 
Beziehungen vorwegnehmen. Solche Arbeiten finden zum einen im Zuge vereinzelter Drittmittelprojekte statt, die auf Initiativen außerhalb der deutschen Planungswissenschaft zurückgehen. Zum anderen sind Qualifizierungsarbeiten ein wichtiges Format zur Erschließung des neuen Themas. Insbesondere im Rahmen von Promotionen entscheiden sich junge Wissenschaftler*innen das neue Thema aufzuschlüsseln: Sie verfügen noch über wenig wissenschaftliches Kapital und sind dementsprechend ungebunden in ihrer Themenwahl. Gleichzeitig suchen sie aktiv nach einem bislang unterrepräsentierten Thema, das potenziell relevant für die Disziplin ist.

Die wissenschaftliche Bearbeitung der neu erkannten Probleme ist originell und fördert Innovationen. Zwar sind nur wenige Planungswissenschaftler*innen mit ihnen befasst und die Verfügbarkeit von Ressourcen ist eingeschränkt, dennoch gelingt es bereits in der Nische, grundlegende Erkenntnisse zu gewinnen. Das neue Wissen muss aus anderen wissenschaftlichen Disziplinen, ausländischen Diskursen, neuen Berechnungen, neuen empirischen Beobachtungen, Gedankenexperimenten und durch deren Verknüpfung mit bekannten Wissensstrukturen geschöpft werden. Das Erschließen des Themas beschreiben einige Pionier*innen dementsprechend als eine Art Puzzle, bei dem erst die Kombination verschiedener Datenquellen ein ,faszinierendes “ Gesamtbild entstehen lässt. Einige Wissenschaftler*innen sind bereits mit besonders passfähigen Herausforderungen und Ideen befasst und können durch geringfügige Modifikationen ihrer Forschungspfade bereits wichtige Erkenntnisse für den Diskurs gewinnen.

\section{Fazit und Phasenübergang}

Zwar wird das Thema in der Latenzphase vereinzelt von Planungswissenschaftler*innen erforscht, die Resonanz bleibt jedoch bis zum Beginn der Durchbruchphase gering. Das akkumulierte Wissen lässt sich dementsprechend nur schwer für Reputation, Drittmittel oder Positionen in Wert setzen. Im Rahmen von Karriereschritten spielt das einschlägige wissenschaftliche Kapital, wenn überhaupt, nur eine nachgeordnete Rolle. $\mathrm{Zu}$ diesem Zeitpunkt ist - auch für die Pionier*innen selbst - noch nicht absehbar, ob, wann und in welcher Form das Thema einen Durchbruch erfahren wird. Wahrscheinlicher als eine disziplinweite Fokussierung ist die anhaltende Existenz in der Nische. Möglich ist auch, dass sich die Pionier*innen nach mehrjährigem Engagement anderen Themen zuwenden und die einschlägige Forschung vollständig abreißt.

In der Latenzphase wurden elementare Erkenntnisse - empirische Befunde, Argumentationen, theoretische Bezüge usw. - der späteren Themenkarriere produziert. Unterschiedliche Facetten des Themas wurden in das Bezugssystem der Raumplanung übersetzt, grundlegende Fragestellungen wurden entwickelt und 
deren Beantwortung in Angriff genommen. Auch ist ein - weitgehend unverbundener - Akteur*innenkreis mit einschlägigen Kompetenzen entstanden. Kommt es zum unmittelbaren Durchbruch, so erfährt dieses bislang unscheinbare epistemische und soziale Kapital eine Neubewertung. In der Retrospektive zeigt sich, dass unmittelbar vor dem Phasenübergang eine Fundierung des Themas stattgefunden hat, indem Inhalte und Akteur*innen vernetzt und in Stellung gebracht worden sind, die im Zuge des Durchbruchs zentrale Bedeutung erlangen.

\subsubsection{Durchbruchphase}

Im gesamten Feld der Raumplanung wird erkennbar, dass die mit dem Thema verbundene Problemstellung von wegweisender Bedeutung ist. Es ist mehr und mehr abzusehen, dass eine Transformation in dem Bereich nicht mehr abzuwenden ist, so dass ein Wettstreit um deren Gestaltung in Gang gesetzt wird. Durch den zunehmend wahrgenommenen Handlungs- und Wissensbedarf gewinnt einschlägiges soziales und epistemisches Kapital schlagartig an Bedeutung. Die Forschungs- und Vernetzungsaktivitäten innerhalb planungswissenschaftlicher Nischen werden nun kombiniert und in das Zentrum des fachgemeinschaftlichen Interesses gehoben. Damit einher geht die Infragestellung etablierter Wissensund Machtstrukturen in der Planungswissenschaft. Es findet ein institutioneller Wandel statt, bei dem das Thema innerhalb von ein bis zwei Jahren von der strukturellen Vernachlässigung und Tabuisierung der Latenzphase zur prioritären Behandlung der Fokusphase aufsteigt.

\section{Institutioneller Kontext}

Es häufen sich Ereignisse, die das Problem auf die massenmediale und politische Agenda rücken lassen. Solche Ereignisse können die Veröffentlichung eines Kommissionsberichts, der Abschluss eines internationalen Abkommens, eine Katastrophe, ein popkulturelles Phänomen und vieles mehr sein. Ein Frame wird etabliert, der die unterschiedlichen Ereignisse und Beobachtungen verknüpft und in dessen Rahmen Handlungsbedarfe ermittelt und potenzielle Lösungen verhandelt werden. Das Thema gelangt immer wieder in den Fokus der medialen Berichterstattung und erhält rasch wachsende Aufmerksamkeit in großen Teilen der Gesellschaft, darunter Akteur*innen der Verwaltung, Politik sowie der Wissenschaft.

Im Zuge der wachsenden gesamtgesellschaftlichen Aufmerksamkeit erkennen Akteur*innen der Planungspraxis und -politik die Relevanz des Themas für ihren jeweiligen Handlungsbereich. Die Aussicht auf zusätzliche Fördermittel und einen Machtgewinn führt zum weitläufigen Eingeständnis von Handlungs- 
und Wissensdefiziten und damit zur Aufhebung der Tabuisierung. Die Zuständigkeit für das Thema ist nun mit einem Bedeutungsgewinn verknüpft und für Entscheidungsträger*innen bietet das Thema eine Gelegenheit, sich und den eigenen Entscheidungsbereich zu profilieren. Akteur*innen verschiedener ministerieller Zuordnungen machen es sich daher zu eigen und bemühen sich, eine treibende Rolle einzunehmen. Das nahezu simultane Aufgreifen des Themas in unterschiedlichen Einrichtungen löst einen selbstverstärkenden Effekt aus: Zum einen entsteht schlagartig ein Resonanzraum, in dem die einschlägigen Kommunikationen der Akteur*innen auf starke gegenseitige Nachfrage stoßen. Zum anderen treten die Akteur*innen zunehmend in Konkurrenz zueinander. Angesichts dieser Dynamik sehen sich politische Akteur*innen unter Zugzwang: Sie müssen zeigen, dass sie die Bedeutung des Themas erkannt haben, dass sie Maßnahmen ergreifen und dass sie eine wichtige Rolle für die Lösung des Politikproblems spielen. Im nun entfachten politischen Wettstreit um Macht und finanzielle Ressourcen benötigen sie umgehend Argumente, um das Thema in ihrem Sinne rahmen zu können. Für die Gestaltung, Umsetzung und Legitimierung von Maßnahmen sind sie auf wissenschaftliches Wissen angewiesen. Es entstehen Handlungs- und Zeitdruck.

Das gesamte Feld der Raumplanung wird in eine Irritation versetzt. Große Wissensbedarfe werden mit zunehmender Dringlichkeit von außen an die Planungswissenschaft herangetragen. Schnell werden Kontakte geknüpft und Kommunikationskanäle etabliert. Schnittstellen und Übersetzer*innen zwischen Wissenschaft und Politik kommt jetzt eine zentrale Rolle zu. Insbesondere Ressortforschungseinrichtungen koordinieren einerseits die Beschaffung des benötigten Wissens für die Politik und organisieren andererseits die Allokation finanzieller Ressourcen sowie die Einflussnahme auf Strategiepapiere, Gesetzesänderungen und Förderprogramme der Planungspraxis für die Wissenschaft. Es schält sich eine Hierarchie, oder zumindest funktionale Ausdifferenzierung, zwischen den einzelnen Kommunikationskanälen heraus: Bestimmte Kontakte, die bereits eingespielt waren oder die sich für bestimmte Fragen als besonders effektiv und effizient erweisen, nehmen vorübergehend eine herausgehobene Makler- bzw. Vermittlerfunktion zwischen Wissenschaft und Politik ein.

In kurzer Zeit werden weithin wahrnehmbare Förderprogramme von Bundesministerien - bei den untersuchten Fallstudien insbesondere BMBF, BMVBS/BMVBW und BMU - sowie deren untergeordneten Behörden angestoBen. Bestehende Förderprogramme und Projekte werden nachträglich um das neue Thema erweitert. Dass die jeweiligen Initiativen sich inhaltlich überschneiden, stellt für die Fördermittelgeber*innen nicht unbedingt ein Hemmnis dar. Sie konkurrieren teilweise darum, frühzeitig einschlägige Förderprogramme zu initiieren, um schließlich Forschungsergebnisse und Problemlösungen vorlegen zu können. 
Weitere Einrichtungen auf Landes-, Regions- und kommunaler Ebene beauftragen Planungswissenschaftler*innen und Planungsbüros, um ebenfalls die Zusammenhänge zwischen dem Thema und dem eigenen Wirkungsbereich zu beleuchten und Handlungsempfehlungen sowie passfähige Strategien zu erhalten.

Durch die zunehmende gesamtgesellschaftliche Aufmerksamkeit und Nachfrage nach einschlägigen wissenschaftlichen Erkenntnissen verändern sich die Anreizstrukturen der Planungswissenschaftler*innen. Zunehmend ist eine besonders schnelle Vermehrung des sozialen, ökonomischen und politischen Kapitals als Belohnung für die einschlägige Forschung zu erwarten. Die epistemische Motivation rückt deshalb gegenüber strategischen Interessen als maßgebliche Antriebskraft für die Beschäftigung mit und die Kommunikation über das Thema in den Hintergrund.

\section{Akteur*innen}

Durch die Verschiebung der Ressourcenallokation zugunsten der einschlägigen Forschung geraten etablierte Machtstrukturen in der Planungswissenschaft unter Druck. Die vor dem Durchbruch mit strukturellen Hemmnissen konfrontierten einschlägigen Forscher*innen werden nun zu gefragten Expert*innen in der Planungswissenschaft, -praxis und -politik. Allein durch die Veränderung des institutionellen Kontextes gewinnen bereits bestehende Kompetenzen und Wissensansprüche der Pionier*innen schlagartig an Wert. Das gleiche gilt für die - jetzt verstärkt in Anspruch genommenen - über Jahre aufgebauten Beziehungen zu weiteren einschlägig forschenden Akteur*innen, deren epistemisches und soziales Kapital ebenfalls an Bedeutung gewinnt. Auf der anderen Seite sehen die in der Disziplin etablierten Akteur*innen, die zuvor die strukturelle Vernachlässigung und Tabuisierung des Themas exekutiert haben, nun ihre wissenschaftliche Autorität in Frage gestellt. Teilweise können sie einen Bedeutungsverlust abwenden, indem sie schnell auf das neue Thema aufsatteln: Noch verfügen sie über den nötigen Einfluss, das Spezialwissen und die Beziehungen, die es ihnen ermöglichen, auch in dem neuen Diskurs eine herausgehobene Position einzunehmen.

Die überwiegend jungen Akteur*innen, die unmittelbar vor dem Durchbruch Ressourcen in die Erforschung des Themas investiert haben, gewinnen angesichts der rasch zunehmenden Resonanz Vertrauen in ihr epistemisches Kapital. Sie verspüren ein erhöhtes Bedürfnis, den Wissensvorsprung - insbesondere in Form von wissenschaftlichen Publikationen - zu kommunizieren und Wissensansprüche geltend zu machen. Es gilt jetzt, Wettbewerbsvorteile in Form themenspezifischer Reputation, Beziehungen und Wissen in Wert zu setzen und für die Zukunft zu sichern.

Weitere Planungswissenschaftler*innen beginnen sich für das Thema, die Pionier*innen und ihre Erkenntnisse zu interessieren. Die Avantgardist*innen erkennen 
die potenzielle Bedeutung des Themas nicht nur für ihren bisherigen Forschungsbereich, sondern auch für die eigene Karriere: Wer mit der eigenen Forschung an das neue Thema anknüpfen kann, verschafft den eigenen Wissensansprüchen eine erhöhte Relevanz. Die Akteur*innen beginnen Zusammenhänge zwischen dem Thema und der eigenen Forschung zu erkunden und machen mit Vorträgen, Publikationen und Fördermittelanträgen auf sich aufmerksam. Das Thema wird so in verschiedene Teilbereiche der Disziplin übersetzt und der einschlägige Akteur*innenkreis erweitert sich. Es setzt ein selbstverstärkender Effekt ein: Planungswissenschaftliche Akteur*innen kommunizieren zunehmend über das Thema, dadurch verdichtet sich der Resonanzboden für einschlägige Forschungsbeiträge, sodass weitere wissenschaftliche Akteur*innen ihren Ressourceneinsatz zugunsten des Themas verlagern. So wie die wachsende Popularität des Themas dazu führt, dass mehr und mehr Akteur*innen verschiedener Fachbereiche das Aufgreifen des Themas im Kontext ihrer Forschung abwägen, so ist das weitläufige Aufgreifen des Themas Grundlage für dessen Diffusion und wachsende Popularität.

Mit den nun in Aussicht stehenden Ressourcen sowie der wachsenden Konkurrenz in der einschlägigen Forschung einher geht eine veränderte Bedeutung von Zeit: Während es in der Latenzphase angesichts der kaum vorhandenen Resonanz und Konkurrenz eine geringe Rolle spielte, wann ein einschlägiger Beitrag veröffentlicht wird, setzt nun eine deutlich erhöhte Dringlichkeit ein, Wissensansprüche zu markieren und Sichtbarkeit in der Fachgemeinschaft zu erlangen. Die Akteur*innen verspüren Druck sich zu positionieren, um Ressourcen generieren zu können.

Die Vernetzungsaktivität nimmt schlagartig Fahrt auf. Akteur*innen, die bereits zu dem Thema publiziert haben, werden zu Wissenshubs und gefragten Kooperationspartner*innen. Gleichzeitig betreten neue Akteur*innengruppen den Diskurs und bringen ihre Kompetenzen und Beziehungen in die Debatten ein. Ministerien und Ressortforschungseinrichtungen nehmen Kontakt zu einschlägig erfahrenen Planungswissenschaftler*innen auf, um einen Überblick über das neue Thema und die fehlende Expertise von ihnen zu erhalten. Arbeitskreise werden konstituiert und verknüpfen Expert*innen verschiedener Teilgruppen des Netzwerks bzw. unterschiedlicher geografischer und institutioneller Zuordnungen miteinander. Erste Workshops und Konferenzen werden einberaumt, die die einschlägig erfahrenen und interessierten Akteur*innen zusammenbringen. Die Auftragsforschungen, Drittmittelanträge, Veranstaltungen und Arbeitskreise führen zu neuen Beziehungen zwischen Akteur*innen aus Planungswissenschaft und -praxis oder erweitern bereits bestehende Beziehungen um einen neuen inhaltlichen Aspekt. Die in der Latenzphase erfolgte, inkrementelle Vernetzung durch die Herausbildung mehrerer unverbundener, einschlägig spezialisierter Teilgruppen, wird durch eine 
sprunghafte Integration verschiedener Teilbereiche des Netzwerks zu einem Mainstreamdiskurs abgelöst. Die gerichtete, persönliche Kontaktaufnahme, bei der das Interesse einer Person durch die Kommunikation einer anderen, bereits einschlägig forschenden Person geweckt wird, ist hierfür nicht mehr allein maßgeblich. Der entscheidende Faktor für die schlagartig erhöhte Netzwerkkohäsion ist stattdessen die Aufnahme von Beziehungen zwischen jeweils bereits einschlägig forschenden Akteur*innen untereinander, sodass diese als Makler*innen ihre jeweiligen Teilgruppen verknüpfen. Hinzu kommen Akteur*innen, für die das Thema zwar neu ist, die aber bereits eine zentrale Position im planungswissenschaftlichen Netzwerk einnehmen. Strukturelle Löcher werden so geschlossen und es bildet sich eine Gigantische Komponente im Ko-Publikationsnetzwerk heraus. Das heißt, bislang unverbundene Teilbereiche des einschlägigen Kooperationsnetzwerks verknüpfen sich zu einer Netzwerkkomponente, die um ein Vielfaches größer ist als die übrigen, nicht mit ihr in Verbindung stehenden Komponenten des Netzwerks (siehe Abb. 9.4).

\section{Inhalt}

Indem das neue Thema auf die Agenda rückt und dessen Tabuisierung aufgehoben wird, wird die Geltung etablierter Erkenntnisse, Instrumente und normativer Grundsätze in einigen Teilbereichen der Disziplin zunehmend in Frage gestellt. Im Sinne Kuhns lässt sich von einer Krise sprechen. Das heißt, die identifizierten Anomalien haben ein solches Ausmaß angenommen, dass das vorherrschende Paradigma nicht mehr aufrecht erhalten werden kann. Der Wettstreit um die Deutungshoheit und die Durchsetzung alternativer Ansätze gewinnt an Fahrt, so dass die Planungswissenschaft einen besonders innovativen und kreativen Moment erlebt. Das Framing - einschließlich der inhaltlichen Tragweite, der institutionellen Zuständigkeit und der einzusetzenden Ressourcen - ist vorübergehend in besonderem Maße formbar. Einen großen Einfluss darauf üben Austauschprozesse im Rahmen hoch angesiedelter Auftragsforschungen und Gutachten, anlaufende Ausschreibungsverfahren von großen Drittmittelprogrammen sowie nun ins Leben gerufene überregionale Arbeitskreise aus. Die Wissenschaftler*innen stellen darin Erkenntnisse bereit, was auch die Formulierung von Wissenslücken miteinschließt. Jetzt entscheidet sich, welche Probleme priorisiert und welche Lösungsoptionen in den kommenden Jahren anvisiert werden. Die Planungswissenschaft ist bei dieser Aushandlung des Framings nicht unbeteiligt, das letzte Wort hat aber die Politik, weil sie durch Förderprogramme, Gesetze und Strategiepapiere über die nötigen Machtinstrumente für dessen Durchsetzung verfügt.

Die inhaltliche Entwicklung der Themenkarriere ist von der Krise und der daraus folgenden Suche nach umgehend verwertbarem Wissen geprägt. Die Fachbeiträge, 
die jetzt produziert werden, leisten primär die grundlegende diskursive Herstellung der Relevanz und die Übersetzung des Themas in das Bezugssystem der Raumplanung. Insbesondere werden jetzt verstärkt bestehende Wissensbestände zusammengetragen und kombiniert, Handlungsbedarfe formuliert und Wissenslücken umrissen. Immer mehr Verknüpfungen zwischen bestehenden Wissensbereichen und dem neuen Thema werden erkennbar. Allerdings findet durch das von politischen Handlungslogiken bestimmte Framing eine temporäre Verengung des Themas auf Teilaspekte statt, für die Lösungsansätze im Feld der Planungspraxis nicht nur dringend benötigt, sondern gleichzeitig auch besonders greifbar und unmittelbar umsetzbar erscheinen. Das heißt, im Rahmen des bestehenden institutionellen Kontextes der Raumplanung verfügbare Steuerungsinstrumente und Strategien sowie die für deren Formulierung und Implementierung benötigten Wissensbestände und deren legitime Sprecher*innen geraten zunehmend in den Fokus.

Für die Markierung einschlägiger Wissensansprüche ist angesichts der entstandenen Krise im gesamten Feld der Raumplanung jetzt ein günstiger Zeitpunkt. Nicht nur kann auf das Framing des Themas in besonderem Maße Einfluss genommen werden, auch ist die Aufmerksamkeit gegenüber neuen Erkenntnissen äußerst hoch. Angesichts der Signale der Fördermittelgeber*innen ist zudem absehbar, dass einschlägige Forschungen weiterhin an Bedeutung gewinnen werden. Es kann deshalb von einer bevorstehenden Erweiterung der einschlägigen Fachgemeinschaft und einer Erhöhung der Publikationszahlen ausgegangen werden, so dass jetzt veröffentlichte Erkenntnisse besonders gute Voraussetzungen für eine weitreichende Rezeption und Zitierung haben. Insbesondere grundlegende Publikationen aus Politik, Praxis oder Wissenschaft, die jetzt systematisch den Wissenstand zum Thema zusammenfassen, Regulierungsoptionen benennen oder die Relevanz des Themas herausstellen, werden in den Folgejahren zu den meistzitierten Publikationen der gesamten Themenkarriere zählen.

\section{Fazit und Phasenübergang}

Im Zuge der Konferenzen, Drittmittelprogramme und Arbeitskreise wird das vorhandene Wissen fachöffentlich zusammengetragen und evaluiert. Es werden - größtenteils mit Beteiligung politischer und planungspraktischer Akteur*innen - Wissenslücken ermittelt und mehrjährige Arbeitsprogramme zu deren Schließung entworfen. Akteur*innen mit bestimmten Spezialisierungen werden zu Expert*innen für das neue Thema und gewinnen wissenschaftliche Autorität. Es wird also innerhalb kurzer Zeit ein über mehrere Jahre hinweg maßgeblicher personeller und inhaltlicher Bezugsrahmen etabliert und mit Ressourcen versehen. Auf diese Weise wird das Thema geframed, das nun eine Fokussierung erfährt. Es ist absehbar, dass Inhalte und Akteur*innen, die Teil dieses Frames sind, in den kommenden Jahren 
in das Zentrum der fachöffentlichen Aufmerksamkeit rücken werden. Dementsprechend suchen zahlreiche Planungswissenschaftler*innen nach Verknüpfungen zwischen dem eigenen Forschungsportfolio und dem neuen Diskurs, was gleichzeitig Ursache und Folge der inhaltlichen und personellen Diffusion bzw. der wachsenden Aufmerksamkeit ist.

Nachdem die Frequenz einschlägiger Publikationen gegenüber der Latenzphase einen raschen Anstieg verzeichnet, markiert ein vorläufiger Höhepunkt der Aufmerksamkeitskurve den Übergang von der Durchbruchphase zur Fokusphase. Angesichts zahlreicher bereits stattfindender Aktivitäten fällt die in Publikationen manifestierte Aufmerksamkeit unmittelbar vor diesem ersten Höhepunkt vergleichsweise gering aus. Dies ist einerseits darauf zurückzuführen, dass nur wenige Planungswissenschaftler*innen über das epistemische Kapital verfügen, um umgehend Wissensansprüche veröffentlichen zu können. Andererseits liegt zwischen der erstmaligen Einreichung eines Manuskripts und der Veröffentlichung des Artikels in der Regel ein Zeitraum von mehreren Monaten. Ein Großteil der zu Beginn der Fokusphase veröffentlichten einschlägigen Artikel wird also bereits in der Durchbruchphase vorbereitet. Die Wissenschaftler*innen visieren für die Veröffentlichung ihrer Erkenntnisse primär die Zeitschriften mit den kürzesten Zeiträumen zwischen Artikelplanung und -veröffentlichung an. Diese weisen gleichzeitig eine hohe Praxis- und Politikorientierung auf, sodass nicht die Einhaltung wissenschaftlicher Standards, sondern die schnelle Anwendbarkeit des Wissens im gesamten Feld der Raumplanung im Vordergrund steht.

\subsubsection{Fokusphase}

Nicht nur ist die Kritik an der Thematisierung verstummt. Aufgrund des klar erkennbaren Handlungs- und Wissensbedarfs ist zudem kaum noch nachvollziehbar, wie das Thema so lange Zeit ignoriert werden konnte. Etablierte Erkenntnisse und Deutungsmuster sind in Frage gestellt worden und ihre Revision setzt sich mehr und mehr durch. Die Entwicklungen der Durchbruchphase - insbesondere die gestiegene Nachfrage nach einschlägigem Wissen, die dadurch stark verbesserte Aussicht auf Reputation und Ressourcen und der wiederum dadurch entfachte Wettbewerb um Aufmerksamkeit und zur Verfügung gestellte Ressourcen - führen zu einer massiven Erhöhung des wissenschaftlichen Outputs. In einem erstaunlich kurzen Zeitraum ist das Thema aus der Nische in das Zentrum des planungswissenschaftlichen Diskurses gerückt. Die Ausschreibungen für die 
großen Förderprogramme, deren Grundlagen in der Durchbruchphase geschaffen wurden, werden jetzt weithin wahrnehmbar veröffentlicht. Die Krise der Durchbruchphase weicht einer Verständigung auf prioritäre Wissens- und Handlungsbedarfe, auf das Vorgehen zu deren Deckung sowie auf den damit befassten Akteur*innenkreis. Es setzt eine nachholende Wissensproduktion ein, bei der ein offensichtlich zu unrecht vernachlässigtes Thema mit Vehemenz in das Zentrum der disziplinären Wissensproduktion gehoben wird, sodass das in der Latenzphase entstandene Defizit durch eine vorübergehende Fokussierung kompensiert wird.

\section{Institutioneller Kontext}

Die Aufmerksamkeit gegenüber dem Thema in Planungspolitik und -praxis ist zu Beginn der Fokusphase hoch. Nachdem das Problem gerahmt, dessen Relevanz hergestellt und Ressourcen verfügbar gemacht wurden, stehen jetzt insbesondere die Politikformulierung, -umsetzung und -evaluierung auf der Tagesordnung. Das Thema wird zu einem prioritär verhandelten Gegenstand politischer Regulierung, was tiefgreifende Transformationen im gesamten Feld der Raumplanung nach sich zieht. Es werden Institutionen - Gesetze, Planungen, Instrumente, Ziele, Ressourcenallokationen - benannt, die vor dem Hintergrund des neuen Themas eine Modifikation erfordern. Finanzmittel, Wissen und Personal werden jetzt nicht mehr nur für die Problemdefinition und Politikformulierung benötigt, sondern auch für die Politikumsetzung.

Vermittelt durch vielfältige strukturelle Kopplungen verändert dieser Wandel die Relevanzkriterien und Anreizstrukturen innerhalb der Planungswissenschaft. So führt bspw. die Umsetzung einschlägiger Förderprogramme im gesamten Feld der Raumplanung zu einer erhöhten Nachfrage nach einschlägiger Expertise, zu veränderten Anforderungsprofilen von Hochschulabsolvent*innen sowie zu einer Transformation des Forschungsgegenstandes, also räumlicher Entwicklungen und deren planerische Gestaltung. In Reaktion darauf werden bspw. Lehrveranstaltungen und Curricula angepasst, in Berufungsverfahren wird einschlägige Expertise honoriert und in Forschungsinstituten werden Schwerpunktsetzungen verschoben. Auch der sprunghafte Anstieg verfügbarer Drittmittel für das Thema im Rahmen allgemeiner Forschungsförderungen, Auftragsforschungen sowie Politikberatungen bewirkt eine planungswissenschaftliche Fokussierung, die sich unter anderem in Stellenausschreibungen, Konferenzen und Publikationen manifestiert. Wurde einschlägige Kompetenz bislang kaum nachgefragt und belohnt, werden nun über mehrere Kanäle Ressourcen für die Erforschung des Themas verfügbar.

Die Krise, die Planungspraxis, -politik und -wissenschaft in der Durchbruchphase über einen kurzen Zeitabschnitt hinweg ergriffen hat, ist zu Beginn der 
Fokusphase einer gewissen Planungssicherheit in Bezug auf inhaltliche, prozedurale und strukturelle Parameter der weiteren Themenkarriere gewichen. Die Wissensbedarfe sind weiterhin hoch, allerdings herrscht jetzt eine gewisse Klarheit, welche Forschungsprobleme für besonders dringlich und wichtig erachtet werden und wie eine Schließung der Wissenslücken zu erreichen ist. Dieses in der Durchbruchphase aufgesetzte Arbeitsprogramm wird in der Fokusphase unter hohem Ressourceneinsatz über mehrere Jahre hinweg abgearbeitet.

Der institutionelle Kontext hat sich gewandelt (siehe Abb. 9.2): Nachdem in der Latenzphase die Zuständigkeit für und die Beschäftigung mit bestimmten Problemstellungen (rot) sanktioniert wurde bzw. andere Themen (grau) bevorzugt wurden, und nachdem in der Durchbruchphase diese Ressourcenallokation in Frage gestellt und neu verhandelt wurde, hat sich in der Fokusphase ein neues Thema in der disziplinären Wissens- und Sozialstruktur integriert und etabliert, das vorübergehend strukturell priorisiert wird, bevor anschließend eine Normalisierung einsetzt.

Institutioneller Kontext (kollektive Wissensproduktion \& Systemumwelt)
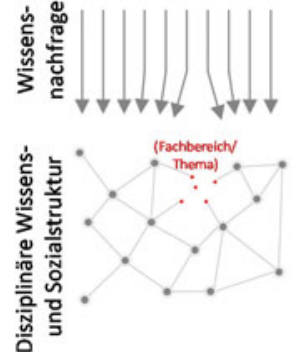

Latenz
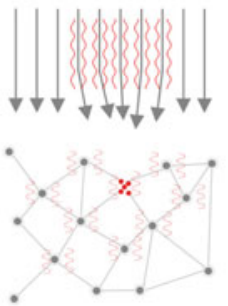

Durchbruch
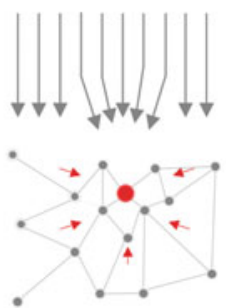

Fokus
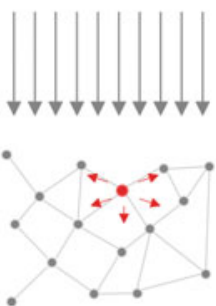

Normalisierung

Abb.9.2 Veränderte Ressourcenallokation gegenüber einem Thema (rot) und ihr Einfluss auf die disziplinäre Wissens- und Sozialstruktur (Quelle: Eigene Darstellung)

Die einschlägigen Förderprogramme in Wissenschaft und Praxis haben großen Anteil daran, dass Planungssicherheit in Bezug auf die Art und den Umfang der Aufwendung fachgemeinschaftlicher Ressourcen für die Erforschung des Themas besteht. Insbesondere die großen Drittmittelprogramme in der Wissenschaft haben im Zuge des Aufstellungsprozesses zunächst die Wissenslücken systematisch unter Einbeziehung verschiedener Akteur*innengruppen erhoben und gerahmt, um diese anschließend durch die Ausschreibungs- und Wettbewerbsverfahren, an denen sich zahlreiche Planungswissenschaftler*innen beteiligen, zu kommunizieren. Es ist 
damit zu rechnen, dass insbesondere die Akteur*innen, die ein Drittmittelprojekt einwerben, in den Folgejahren als Konferenzteilnehmer*innen und Autor*innen in dem einschlägigen Diskurs in Erscheinung treten. Sie haben sich nicht nur durch ihre vielversprechenden Ideen, ihre Beziehungen und ihre Kompetenzen in einem Bewerber*innenfeld durchgesetzt. Auch ist durch die im Rahmen des Programms abgerufenen finanziellen Ressourcen sowie die vorgesehenen Veranstaltungen, Publikationen und Evaluierungen mit einem Mindestmaß an wissenschaftlichem Output, personeller Verstärkung und Vernetzung zu rechnen. Dies führt zu einer über mehrere Jahre hinweg stark erweiterten themenbezogenen Fachgemeinschaft, die nicht nur neue einschlägige Erkenntnisse erwarten lässt, sondern auch die Resonanz darauf gewährleistet.

\section{Akteur*innen}

Strukturelle Anreize und Hemmnisse für die wissenschaftliche Themenwahl geben insbesondere zu Beginn der Fokusphase einschlägigen Forschungsaktivitäten Vorrang gegenüber anderen Themen. Um den Zugang zu Fördermitteln sicherzustellen, die Relevanz der eigenen Arbeiten zu unterstreichen und die Resonanz auf eigene Wissensansprüche zu erhöhen, ist es daher jetzt für Wissenschaftler*innen von Vorteil, das eigene Forschungsprogramm mit dem neuen Thema diskursiv zu verknüpfen. Drittmittelanträge, Arbeitskreise, Publikationen und Vorträge werden zum Anlass genommen, einschlägiges Wissen zu produzieren, vorhandene Wissensbestände auf Anschlussfähigkeit zu überprüfen und erworbene Erkenntnisse zu verwerten. Auch sind sie Katalysatoren für neue Kooperationen und die inhaltliche Erweiterung bestehender Kooperationen, so dass sich das themenbezogene Netzwerk gleichzeitig vergrößert und verdichtet.

Die entstandene Planungssicherheit manifestiert sich auch in den Akteur*innenkonstellationen. Das heißt, aufgrund der allgemein anerkannten Relevanz des Themas hält die einschlägige Forschung - anders als bisher - langfristige Karriereoptionen bereit. Die Hektik bzw. der Zeitdruck, unter dem die Akteur*innen in der Durchbruchphase vorhandenes Wissen kommuniziert haben, weicht nach Aufnahme der Drittmittelprojekte einer langfristigen und systematischen Produktion neuen Wissens. Die Verringerung einschlägiger Artikelzahlen unmittelbar nach dem ersten Aufmerksamkeitshoch zu Beginn der Fokusphase lässt sich auf das Einsetzen dieser mehrjährigen Produktionsphase zurückführen.

Die einschlägige Fachgemeinschaft differenziert sich nicht nur horizontal entlang neuer Spezialgebiete aus, sondern auch vertikal anhand des sozialen, ökonomischen und politischen Kapitals. Im Sinne von Cranes (1969b) „,core and scatter“ gibt es jetzt gut sichtbare Autoritäten, die besonders stark an Reputation und Einfluss gewinnen. Sie geben neu hinzukommenden Wissenschaftler*innen Orientierung 
und gewährleisten, dass deren wichtige Erkenntnisse nicht verloren gehen, sondern rezipiert und akkumuliert werden (siehe Kp. 2.3). Einige Pionier*innen, die bereits in der Nische zentrale Positionen einnahmen, üben nun führende Funktionen in der einschlägigen Politikberatung, Drittmittelforschung und Wissensproduktion aus. Indem sie jetzt deutlich mehr zitiert werden, ihre Kooperationsbeziehungen an Prestige gewinnen und sie erfolgreich Forschungsprojekte einwerben, erhöhen sich ihre Reputation, ihre finanziellen Ressourcen sowie ihr Einfluss. Trotz der weiterhin teils starken Spezialisierung ihres Forschungsportfolios entwickeln sie sich also mit dem Durchbruch des Themas von Teilnehmenden eines peripheren Teildiskurses zu zentralen Akteur*innen der Disziplin.

Auch einem Teil der Avantgarde, die im Zuge der Durchbruchphase das Thema aufgegriffen hat, gelingt es, ihre wissenschaftliche Autorität durch einschlägige Forschungsaktivitäten zu erweitern. Voraussetzung hierfür ist, dass die Akteur*innen bereits vor der Durchbruchphase über ein gewisses Maß an wissenschaftlichem Kapital verfügt haben. Gelingt es den Avantgardist*innen im Zuge der Durchbruchphase ihr Forschungsportfolio erfolgreich mit dem neuen Thema zu verknüpfen und so den Diskurs mit Beziehungen und Wissen zu bereichern, werden sie Teil des über Systemgrenzen hinweg sichtbaren Expert*innenkreises. Auch sie werden für Forschungs- und Beratungsaufträge, wissenschaftliche Kooperationsanfragen, Zitationen usw. in Betracht gezogen. Sie erweitern ihr ökonomisches und politisches Kapital und gewinnen im wissenschaftlichen Netzwerk an Zentralität (siehe Abb. 9.4).

Im Zuge der zahlreichen anlaufenden Drittmittelprojekte und der dadurch erfolgenden Stellenbesetzungen beginnen sich viele, insbesondere junge Planungswissenschaftler*innen erstmals systematisch mit dem Thema $\mathrm{zu}$ befassen. Im Rahmen der Förderprogramme haben sie vereinfachten Zugang zu einschlägigem Wissen und Netzwerken. Allerdings ist es in der mittlerweile großen einschlägigen Fachgemeinschaft schwieriger geworden, Sichtbarkeit zu erlangen, zumal die jungen Forscher*innen kaum über wissenschaftliches Kapital verfügen. Sie zählen zu den Anwender*innen und müssen sich zunächst den Pionier*innen und den Avantgardist*innen unterordnen. Im Rahmen von Angestelltenverhältnissen führen sie vorab definierte Forschungsprogramme aus und verschaffen ihren Vorgesetzten Wissen und Einfluss. In der fachgemeinschaftlichen Wissensproduktion nehmen sie weniger reputationsträchtige Funktionen wahr, wie etwa die empirische Fundierung bereits bestehender Wissensansprüche. Durch Konferenzteilnahmen, Zitationen und die Anwendung von Erkenntnissen schaffen sie einen dichten Resonanzboden insbesondere für die Kommunikationen jener Akteur*innen, die bereits über einen Vorsprung in Bezug auf ihr wissenschaftliches Kapital in dem Themenfeld verfügen. Bevor sie eigene Wissensansprüche 
markieren können, müssen sie den mittlerweile umfangreichen Wissenskanon aufarbeiten und darin eine eigene Nische finden. Im einschlägigen Netzwerk sind sie in gewissem Maße von Gatekeeper*innen abhängig und auf die Auskunfts- und Kooperationsbereitschaft etablierter Akteur*innen angewiesen. Über die im Zuge der Fokusphase neu hinzugekommenen Wissenschaftler*innen resümiert eine*r dieser Gatekeeper*innen:

„Ich wüsste jetzt nicht, dass [...] richtig neue Namen nochmal dazu gekommen sind,

[...] wo ich sagen würde, die haben eine größere Wahrnehmung erreicht. "(i67)

Indem viele Planungswissenschaftler*innen das neue Thema aufgreifen, diffundiert das Thema in die verschiedenen Teilbereiche der Disziplin und verändert individuelle und kollektive Wissensstrukturen. Die Akteur*innen verknüpfen ihre jeweiligen Forschungsportfolios mit dem für sie neuen Thema, was sich unter anderem darin zeigt, dass sie in hohem Maße besonders aktuelle Publikationen sowie verstärkt eigene Veröffentlichungen zitieren. Durch das Einbringen ihrer jeweiligen Forschungsbiografien, erweitern die Akteur*innen den Diskurs um ihre jeweiligen Interessen, Themen, Theorien, Methoden, regionalen Schwerpunkte und persönlichen Beziehungen. Die Akteur*innen erschließen so das neue Thema für ihre jeweiligen Fachbereiche und schaffen neue Fragestellungen und Anknüpfungspunkte für wiederum neue Wissenschaftler*innen. Mit dieser Diffusion einher geht die inhaltliche Verschiebung und Ausdifferenzierung des einschlägigen Diskurses.

Aber nicht nur das Thema wird durch dieses Prinzip verändert und erweitert, die Themenkarriere modifiziert umgekehrt auch die individuellen Forschungsbiografien maßgeblich und nachhaltig. Durch die Verknüpfung ihrer bisherigen Forschungspfade mit dem Thema werden die Akteur*innen und ihre Inhalte aus ihren gewohnten Kommunikationszusammenhängen gelöst und für eine größere oder zumindest veränderte Fachgemeinschaft sichtbar gemacht. Zum Thema passende Aspekte der jeweiligen Forschungsportfolios werden von den Akteur*innen vorübergehend in den eigenen Forschungsentscheidungen priorisiert und hervorgekehrt. Durch die neuen Erkenntnisse, Erfahrungen und Kontakte im Kontext der einschlägigen Forschung verändert sich das wissenschaftliche Kapital der Akteur*innen, was sich wiederum auf zukünftige Forschungsentscheidungen auswirkt.

\section{Inhalt}

Die Fokusphase beginnt mit einem vorläufigen Allzeithoch der Häufigkeit einschlägiger Artikel. Gleichzeitig rückt innerhalb der einschlägigen Artikel das neue Thema, nachdem es zuvor primär als Teilaspekt anderer Themen verhandelt wurde, in den Mittelpunkt der Betrachtung. In den Zeitschriften dominieren zu Beginn der 
Fokusphase weiterhin theoriebasierte Beiträge zum Thema, die in die Thematik einführen und zentrale Fragestellungen formulieren. Bestehende Wissensbestände werden mit dem Thema verknüpft, wodurch wiederum rasch neue Erkenntnisse entstehen. Die Artikel sind geprägt von dem Anspruch, das Thema grundsätzlich für die Disziplin und ihre verschiedenen Fachbereiche zu übersetzen, das heißt, aufzuschlüsseln, inwiefern das Thema für die Raumplanung relevant ist, sowie umgekehrt darzustellen welche Rolle der Raumplanung im Kontext der neuen Problemstellung zukommt. Die Titel der Artikel - bspw. „Herausforderung [neues Thema]“, „,[Neues Thema] und Raumplanung“ oder „,Raumplanung: In Zeiten [des neuen Themas] wichtiger denn je" - verdeutlichen diesen Anspruch.

Auch wenn die Wissensdefizite in Planungswissenschaft, -politik und -praxis gleichermaßen erkannt worden sind, wird das erste vorläufige Allzeithoch in der Fokusphase primär getragen durch die vergleichsweise planungspraktisch und -politisch orientierten Zeitschriften PLANERIN, RaumPlanung und IzR. Bei diesen Zeitschriften nehmen die Redaktionen eine stärkere inhaltliche Lenkungsfunktion wahr, im Rahmen derer Praxistauglichkeit und Aktualität zu den wichtigsten Selektionskritieren gehören. Zudem ist der zu betreibende Aufwand und damit der benötigte Zeitraum bis zur Veröffentlichung eines Beitrags geringer als in den wissenschaftlich orientierten Zeitschriften, die stärkeren Wert auf methodische und theoretische Fundierung sowie auf das peer review-Verfahren legen. Dies ermöglicht einerseits den Pionier*innen auf Basis ihres bereits erworbenen epistemischen Kapitals, die plötzlich überall wahrgenommenen Wissensdefizite in kompakter Form zu bedienen und Wissensansprüche schnell geltend zu machen. Andererseits können Akteur*innen, die das Thema jetzt erst aufgreifen, hier Sichtbarkeit erlangen, indem sie Aspekte des neuen Themas im Format eines Gedankenspiels mit einem eigenen Forschungspfad - bspw. eine bestimmte Region, ein disziplinäres Spezialgebiet oder eine Methode - verknüpfen.

Neben der kurzfristig angelegten Veröffentlichung von Wissensansprüchen auf der Grundlage bereits bestehender Wissensbestände nehmen mit Einsetzen der bereits erwähnten Planungssicherheit jetzt auch (wieder) aufwändige, langfristig angelegte Forschungen mehr Raum ein. Sie finden im Rahmen der mehrjährigen Drittmittelprojekte, Arbeitskreise und Qualifizierungsarbeiten statt und resultieren in einem im Laufe der Fokusphase verstärkten Aufkommen einschlägiger Veröffentlichungen in den aufwändigeren Publikationssegmenten. Das heißt, nicht nur ist anhand der Zeitschriften RuR und ARL-FB ein zunehmender Output nachweisbar, auch in Monografien und Sammelbänden dürfte das Thema bis zum Ende der ca. fünfjährigen Fokusphase mehr und mehr Raum einnehmen.

Es setzt das Mainstreaming des Themas ein. Das heißt, das Thema, das zuvor ausschließlich in einer Nische verhandelt wurde, entfaltet sich innerhalb kurzer 
Zeit in verschiedene Bereiche der Planungswissenschaft. An dieser Stelle zeigt sich das integrative Potential des Themas: Je breiter das Spektrum an Normen, Theorien, Instrumenten, Methoden und Fachbereichen, die durch das Thema tangiert werden, desto weitreichender ist die Aufmerksamkeit, die dem Thema entgegengebracht werden kann. Umgekehrt führt die erhöhte Aufmerksamkeit dazu, dass in mehr und mehr Fachbereichen inhaltliche Schnittmengen mit dem Thema geprüft werden. Bereits bekannte kognitive Bezüge zwischen bestehenden Wissensbereichen und dem neuen Thema gewinnen an Bedeutung und weitere Verknüpfungen werden erkannt. Die temporäre Verengung des Themas auf wenige, förderfähige Teilaspekte, die im Zuge des Durchbruchs in den Fokus rückten, weicht zunehmend einer Ausdifferenzierung des einschlägigen Diskurses.

Diese Ausdifferenzierung hängt auch mit der Evolution des Themas im Feld der Planungspraxis zusammen. Nicht nur wird die Dimension des Themas im Rahmen beobachteter räumlicher und gesellschaftlicher Entwicklungen zunehmend sichtbar bzw. wahrgenommen, auch wird die Regulierung des Politikproblems zunehmend formuliert und implementiert. Umsetzungswissen - bspw. über Strukturen, Prozesse, Instrumente und Handlungshemmnisse - gewinnt gegenüber normativen, inhaltlichen und theoretischen Grundlagen an Bedeutung. Die Implementierung von Förderprogrammen, Gesetzesänderungen und angepassten Planwerken zieht außerdem die Veränderung räumlicher und gesellschaftlicher Strukturen nach sich. In den Publikationen rücken daher Fallbeispiele und Evaluationen von Politikansätzen in den Vordergrund. Zunehmend werden Probleme und Interessenkonflikte der neuen Politikansätze offengelegt und Änderungsbedarfe angemeldet. Auch werden kritische Stimmen laut, die die thematische Fokussierung an sich in Zweifel ziehen.

\section{Fazit und Phasenübergang}

Der Beginn der Fokusphase war durch eine große Zahl grundlegender politik- und praxisorientierter Veröffentlichungen geprägt, die als schnelle Reaktion auf den hohen Handlungs- und Wissensbedarf angelegt waren. Hierbei sind grundlegende Fragen und Lösungsansätze formuliert worden, die in einer ca. drei Jahre andauernden Arbeitsphase erforscht wurden. Die Publikationszahlen reichen in diesen drei Jahren nicht ganz an das erste Aufmerksamkeitshoch heran, übertreffen dieses aber zum Ende der Fokusphase und erreichen ein Allzeithoch (siehe Abb. 9.3). Während zum ersten Veröffentlichungshöhepunkt einführende, übersetzende, eher hypothetische Beiträge - häufig nach dem Muster , Was bedeutet [das Thema] für die [Bindestrichplanung]?" - dominierten, rücken im zweiten Höhepunkt empirische Fallstudien und anwendungsorientierte Forschungen in den Fokus.

Der zweite Peak der Aufmerksamkeitskurve ist zu einem großen Teil auf das Auslaufen der drei Jahre zuvor initiierten Arbeitskreise und Drittmittelprogramme 


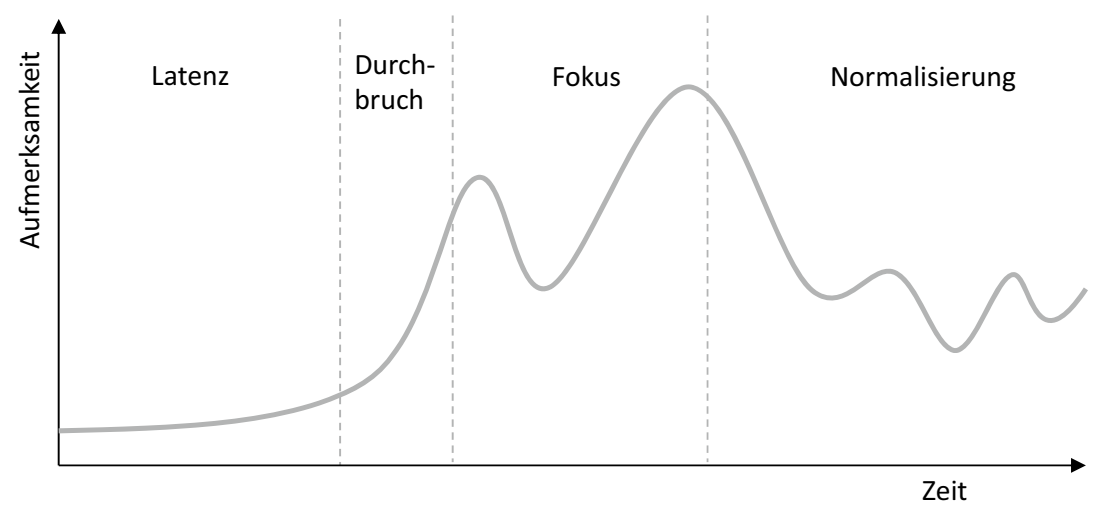

Abb.9.3 Idealtypische Themenkarriere auf Basis der beiden untersuchten Fallbeispiele (Quelle: Eigene Darstellung)

zurückzuführen. Die darin vorgesehenen finanziellen und organisatorischen Unterstützungsleistungen sind mit der - mehr oder weniger formalisierten - Erwartung verbunden, dass Ergebnisse produziert und veröffentlicht werden. Nachdem also zu Beginn der Projekte die verantwortlichen Akteur*innen benannt, die Forschungsfragen festgelegt und die wissenschaftlichen Mitarbeiter*innen eingestellt worden sind, beginnt zunächst eine Arbeitsphase von zwei bis vier Jahren. Nach dieser Phase muss das produzierte Wissen veröffentlicht werden.

In diesem Allzeithoch ist der Übergang zur Normalisierungsphase angelegt. Die zum Ende der Fokusphase publizierten Erkenntnisse erreichen eine bis dahin unerreichte methodische, empirische und theoretische Fundierung. Gleichzeitig sind aber auch eine gegenüber der Durchbruchphase erhöhte Redundanz des produzierten Wissens sowie verringerte Kreativität und Innovativität zu verzeichnen. Mittlerweile hat der Diskurs einen einschlägigen Wissenskanon und zentrale Akteur*innen etabliert, die unter den Diskursteilnehmer*innen als bekannt vorausgesetzt werden können und auf deren Erkenntnisse zahlreiche neue Studien aufbauen. Die zentralen Wissensansprüche sind bereits veröffentlicht worden. Das heißt, die neu erscheinenden Publikationen beschäftigen sich primär mit der Anwendung, Fundierung und Kritik dieser Innovationen.

Wer jetzt noch eine grundlegende Aufarbeitung der Thematik vorlegt, für den*die sollte die Hoffnung auf erhöhte Resonanz kein Antriebsfaktor sein. Nicht nur werden jetzt die meisten Fachbeiträge veröffentlicht, auch veröffentlichen nun mehr planungswissenschaftliche Akteur*innen als je zuvor zu dem Thema. Die Dichte 
des Netzwerks einschlägig publizierender Akteur*innen erreicht ein Maximum, da ab jetzt wichtige Vernetzungsformate (Drittmittelprojekte, Arbeitskreise) auslaufen und sich der Diskurs inhaltlich zunehmend ausdifferenziert. Das Thema vereint nun zahlreiche Teildiskurse, das heißt hinreichend homogene, dynamische Kommunikationszusammenhänge von Akteur*innen, in denen kollektiv ein jeweils spezifisches Wissen produziert wird. Die schnelle und persönliche Kommunikation über neue Erkenntnisse und priorisierten Forschungsbedarf im Kontext des übergreifenden Themas ist kaum mehr möglich und wird nicht mehr angestrebt. Auch inhaltlich ist deshalb ein Maximum an Kohäsion überschritten.

\subsubsection{Normalisierungsphase}

Im zweiten Aufmerksamkeits-Peak ist bereits die anschließende Ermüdung angelegt. Zwar ist durch die stark expandierte, einschlägige Fachgemeinschaft ein verdichteter Resonanzboden entstanden, gleichzeitig ist das Thema aber auch kein Alleinstellungsmerkmal mehr. Im Verhältnis zur Größe der einschlägigen Fachgemeinschaft ist die Bedeutung des verbliebenen und neu identifizierten Forschungsbedarfs gering, weshalb die Konkurrenz um potenzielle Wissensansprüche groß ist. Auch die Verfügbarkeit von Ressourcen und neuen Positionen ist in dem Themenfeld rückläufig. Die Akteur*innen wenden sich verstärkt Detailfragen und anderen Themen zu, so dass sich die fachöffentliche Aufmerksamkeit sukzessive verlagert. Die Zahl der einschlägigen Veröffentlichungen nimmt jetzt in Wellen ab, sie bleibt aber im Vergleich zur Latenzphase auf einem höheren Niveau.

\section{Institutioneller Kontext}

Die hohe Bedeutung des Themas in verschiedenen Bereichen der Planungspolitik, -praxis und -wissenschaft ist unvermindert anerkannt. Es hat sich mittlerweile in Gesetzen, Leitbildern, Plänen, Handlungsprioritäten und Netzwerken manifestiert. Diese Institutionalisierung zieht bundesweit und ebenenübergreifend Umsetzungserfordernisse und damit eine anhaltend hohe Nachfrage nach einschlägiger Expertise im Feld der Planungspraxis nach sich. Auch entsteht neuer Forschungsbedarf in der Evaluation und Optimierung umgesetzter Maßnahmen sowie in der Analyse von Handlungshemmnissen bei mangelhaft oder nicht implementierten Ansätzen im Feld der Raumplanung.

Dennoch trifft zu Beginn der Normalisierungsphase das beispiellos hohe Angebot an neuem, fundiertem wissenschaftlichen Wissen $\mathrm{zu}$ dem Thema auf einen 
stagnierenden bzw. teilweise sinkenden Wissensbedarf außerhalb der Planungswissenschaft. Zahlreiche und umfassende Problemlösungen im Feld der Raumplanung sind entwickelt und umgesetzt worden, so dass die Politik - unabhängig davon, ob das Problem tatsächlich gelöst wurde - sich anderen Fragen zuwendet. Die anhaltend hohe Nachfrage aus der Praxis nach einschlägiger Expertise - bspw. im Rahmen von beauftragten Gutachten, Konzepten oder Entwürfen - lässt sich weitgehend im Rahmen von Handlungsroutinen und allgemein bekannten Vorgehensweisen bedienen. Weiterer Bedarf nach grundlegenden wissenschaftlichen Innovationen wird deshalb immer seltener freigelegt. Im Gegensatz zur Durchbruchphase, in der ein extremer Nachfrageüberhang nach einschlägigen Erkenntnissen vorlag, lässt sich jetzt teilweise von einem Angebotsüberhang in der Kommunikation wissenschaftlicher Erkenntnisse an die Systemumwelt sprechen.

Es ist nunmehr ein Rückgang oder zumindest kein weiteres Wachstum der Aufmerksamkeit gegenüber dem Thema in der Planungswissenschaft zu erwarten. Es wird deutlich, dass der hohe Ressourceneinsatz und die Dominanz des Diskurses innerhalb der Disziplin kaum mehr steigerungsfähig sind. Zunehmend wird Kritik an dieser Fokussierung bzw. der Vernachlässigung anderer Themen laut. Die großen einschlägigen Arbeitskreise und Förderprogramme sind abgeschlossen und die in diesem Kontext zu Beginn der Fokusphase festgelegten Fragen sind zumindest aus Sicht der Beteiligten - sonst müssten sie ihr Scheitern eingestehen - beantwortet. Eine Neuauflage ist daher ausgeschlossen bzw. nur mit Modifikationen und unter anderer Überschrift möglich. Die im Rahmen der einschlägigen Erkenntnisproduktion neu ermittelten Wissenslücken haben nicht mehr die hohe Bedeutung, die der zu Beginn der Fokusphase identifizierte Forschungsbedarf hatte. Die Fokussierung und Planungssicherheit der Vorjahre weichen der Suche nach neuen, verheißungsvollen Wissensbereichen. Während das Interesse an dem übergeordneten Diskurs nachlässt, gewinnen Detailfragen an Aufmerksamkeit, denen noch Wachstumspotenzial zugesprochen wird.

Der abnehmende Innovationsbedarf, der Rückgang von Fördermitteln und die Perspektive der stagnierenden oder rückgängigen Bedeutung der einschlägigen Forschung wirken sich auf das Anreizsystem der Planungswissenschaftler*innen aus. Nicht nur für das Einwerben finanzieller Ressourcen herrschen jetzt vergleichsweise ungünstige Bedingungen, auch für die fachinterne Akkumulation von Reputation oder das Einnehmen einflussreicher Positionen hat das Themenfeld an Attraktivität verloren. Gleichzeitig bringen die Arbeitskreise, Drittmittelprojekte und Qualifizierungsarbeiten der letzten Jahre eine große Zahl einschlägiger Expert*innen hervor. Die Konkurrenz um potenzielle Wissensansprüche, finanzielle Ressourcen und Einfluss ist deshalb in dem Themenfeld intensiver als in anderen Fachbereichen der Disziplin. 


\section{Akteur*innen}

Parallel zu den veränderten Anreizstrukturen stellt sich bei den einschlägigen Expert*innen eine Sättigung in Bezug auf ihre eigene Forschung zu dem Thema ein. Das heißt, sie gewinnen das Gefühl, genug in dem Themenbereich geforscht zu haben und der - gemäß retrospektiver Schilderungen ,,intrinsische“ oder epistemisch motivierte - Wunsch wird größer, jetzt neue Wissensbereiche zu erschließen. Diese Ermüdung auf individueller Ebene wird in ähnlicher Form von den Akteur*innen geschildert, die sich in der Latenzpase von dem Thema abwenden. Sie tritt also immer dann auf, wenn die Resonanz für die einschlägige Forschung vergleichsweise gering ausfällt, und ist unabhängig von einem vermeintlich objektiven Forschungsbedarf. Angesichts des mittlerweile erworbenen themengebundenen wissenschaftlichen Kapitals fällt es einigen einschlägig etablierten Akteur*innen trotz dieser Ermüdung schwer, das Thema ruhen zu lassen. Sie haben zwar kein gesteigertes Interesse, sich noch weiter mit dem Thema zu profilieren, allerdings profitieren sie in dem einschlägigen Diskurs nach wie vor besonders stark von ihren Beziehungen und ihrem Wissen. Immer wieder ergeben sich günstige Gelegenheiten, um mit geringem Aufwand wissenschaftliche Beiträge zu platzieren, renommierte Positionen einzunehmen oder Drittmittel einzuwerben.

Das Thema hat sich in die Forschungsbiografien eingeschrieben. Das heißt, ein Großteil des Wissens, der Beziehungen und der Reputation, über das diese Akteur*innen verfügen, wurde durch einschlägige Forschung erzeugt und in diese reinvestiert. Das von den Akteur*innen im Kontext der Themenkarriere aufgebaute epistemische und soziale Kapital entfaltet nachhaltige Wirkung auf die individuelle und fachgemeinschaftliche Wissensproduktion, indem es Grundlage zukünftiger Forschungsentscheidungen ist. Insbesondere Akteur*innen, die im Vorfeld der Erforschung des Themas über geringes wissenschaftliches Kapital verfügten, sind jetzt vergleichsweise stark auf das Thema festgelegt. Einige Pionier*innen des Themas, also die Akteur*innen, die zum Zeitpunkt des Durchbruchs bereits wissenschaftliches Kapital - insbesondere Wissen, Reputation und Beziehungen - in dem Feld erworben hatten, konnten ihre eigene Karriere bspw. durch vielzitierte einschlägige Veröffentlichungen fördern und bekleiden mittlerweile Professuren, leiten Institute oder eigene Planungsbüros. Das soziale Kapital der Akteur*innen hat sich stark erhöht, nicht nur weil durch die Resonanz auf die einschlägige Forschung Reputation und wertvolle Kontakte hinzugekommen sind, sondern auch, weil die ehemals in der Nische verorteten persönlichen Beziehungen nun ebenfalls stark an Einfluss und Reputation gewonnen haben.

Es werden neue Verwertungsmöglichkeiten für das im Zuge der einschlägigen Forschung erworbene wissenschaftliche Kapital gesucht. Hierbei werden zum einen Diskurse mit thematisch hohen Schnittmengen ins Auge gefasst, die ein höheres 
Innovations- und Reputationspotenzial bieten. Erneut tritt ein selbstverstärkender Effekt ein: Strukturelle Anreize geben anderen Themen Vorzug, weshalb sich mehr und mehr Planungswissenschaftler*innen von dem , alten " Thema abwenden. Dadurch schrumpfen die einschlägige Fachgemeinschaft und die Resonanz auf einschlägige Erkenntnisse, was wiederum eine Veränderung des Anreizsystems darstellt. Zum anderen wird der Fokus auf den internationalen Diskurs verschoben, denn nicht nur bieten international vergleichende Studien weiterhin Innovationspotenzial, auch haben die im deutschsprachigen Diskurs etablierten Erkenntnisse hier teilweise Neuigkeitswert. Sie lassen sich also ein zweites Mal in Wert setzen, jetzt mit einer stark vergrößerten Leserschaft. Zusätzliche Verwertungsmöglichkeiten bieten sich im Feld der Planungspraxis. Hier ist die Nachfrage nach einschlägiger Expertise aufgrund der umfassenden Institutionalisierung des Themas in Gesetzen, Plänen und Förderprogrammen weiterhin hoch, weshalb sich die erworbenen einschlägigen Kontakte, Kompetenzen, Referenzen und Routinen hier weiterhin gut in finanzielle Ressourcen und einflussreiche Positionen eintauschen lassen. Einige Planungswissenschaftler*innen verlagern ihre einschlägigen Aktivitäten daher stärker auf ihr Planungsbüro, andere verlassen die Wissenschaft und wechseln ganz in die Verwaltung oder in die Privatwirtschaft.

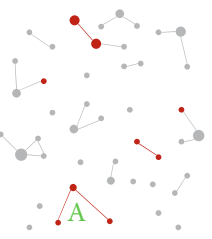

Latenzphase

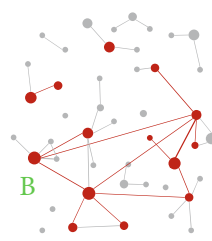

Durchbruchphase

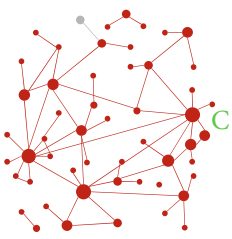

Fokusphase

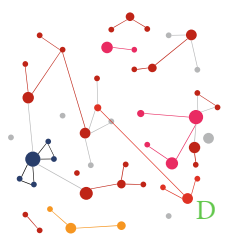

Normalisierungsphase

Abb.9.4 Vernetzung wissenschaftlicher Akteur*innen im Laufe einer Themenkarriere (Quelle: Eigene Darstellung)

Abb. 9.4 stellt die Vernetzungsaktivität im einschlägigen Netzwerk im Zuge der Themenkarriere dar. Alle dargestellten Knoten (Wissenschaftler*innen) sind in mindestens einer der Phasen schwerpunktmäßig mit dem Thema befasst (dann in rot dargestellt). Die Kanten stellen aktive Kooperationsbeziehungen (bspw. Ko-Publikationen, gemeinsame Teilnahme in Arbeitskreisen, gemeinsame Drittmittelprojekte) zwischen den Personen dar. Die Größe der Knoten spiegelt das soziale Kapital der Akteur*innen bzw. die Zentralität im wissenschaftlichen Netzwerk wider. In der Latenzphase gibt es nur wenige verstreute isolates und Teilgruppen, die das Thema bearbeiten (A). In der Durchbruchphase greifen einige Akteur*innen 
das Thema auf, deren bisherige Forschung sich gut mit dem aufkommenden Thema verknüpfen lässt. Einige von ihnen hatten vor ihrer einschlägigen Forschung bereits zentrale Positionen inne (B). Im Zuge einschlägiger Konferenzen und Arbeitskreise werden Beziehungen aufgenommen, die einige Teilgruppen zu einer Gigantischen Komponente zusammenschließen und den direkt verbundenen Akteur*innen (Makler*innen) einen Bedeutungszuwachs verleihen. In der Fokusphase werden einige Akteur*innen, insbesondere durch Stellenbesetzungen im Zuge einschlägiger Projekte, erstmals Teil der Planungswissenschaft. Sie sind in der Hierarchie ihren Vorgesetzten nachgeordnet und verleihen diesen einen Bedeutungszuwachs. Die Pionier*innen (bereits in der Latenzphase aktiv) und Avantgardist*innen (seit der Durchbruchphase aktiv) machen teilweise mit dem Thema Karriere und nehmen mitunter zentralere Positionen ein als ihre (ehemaligen) Vorgesetzten und Mentor*innen (C). In der Normalisierungsphase zerfällt das einschlägige Netzwerk. Einige Akteur*innen gehen in die Praxis oder in den Ruhestand und die Knoten fallen weg. Andere Akteur*innen wenden sich Subthemen zu, die nun eine eigene Themenkarriere erfahren können (D).

\section{Inhalt}

Die im Zuge der Durchbruchphase formulierten wichtigsten Fragen gelten als beantwortet, so dass keine bahnbrechenden Innovationen mit direkter Praxisrelevanz mehr zu erwarten sind. Es werden Ressourcen frei, um auch die zunächst als weniger dringlich erachteten Fragen ins Auge zu fassen, so dass das Thema weiter diffundiert. Fokussiert werden beispielsweise Analysen weniger betroffener Regionen, internationale Vergleiche oder die Aktualisierung von Theoriebeständen. Zudem verlagert sich die Aufmerksamkeit weiter auf die Umsetzung von Politikansätzen, also auf Verfahrensfragen und Wirkungsanalysen. Dabei kommt zunehmend Kritik an den Politikansätzen in Bezug auf ihre negativen Effekte und ihre thematische Einseitigkeit auf.

Das Thema ist nun in den planungswissenschaftlichen Wissenskanon integriert. Das heißt, die grundsätzlich hohe Bedeutung des Themas für die Raumplanung sowie zahlreiche inhaltliche Bezüge zu etablierten Wissensbereichen sind diskursiv hergestellt worden und werden auch im Zuge der nachlassenden Aufmerksamkeit in der Normalisierungsphase nicht mehr in Frage gestellt, sondern erweitert. Neue Diskurse müssen an diesen Wissenskanon anknüpfen, sich also auch gegenüber dem mittlerweile etablierten Thema in Beziehung setzen. Das Thema wird so innerhalb der Disziplin zum unhinterfragten Kontext für die Erforschung neuer Wissensbereiche. 
Bis dato als Subthemen innerhalb des Diskurses wahrgenommene Debatten rücken stärker in das Zentrum der Aufmerksamkeit, und sie erfahren eigene Themenkarrieren. Die thematische und begriffliche Verschiebung ist das Resultat einer nötig gewordenen Differenzierung innerhalb eines kaum mehr überschaubaren Diskursfeldes. Sie dient also einerseits der Abgrenzung und Verdichtung von Kommunikationszusammenhängen. Den (ehemaligen) Subthemen wird aber gleichzeitig auch ein größeres Innovationspotenzial zugesprochen, während die wichtigen Fragen des ,alten" Themas als weitgehend erforscht gelten. Diese Sichtweise wird von den Fördermittelgeber*innen gestützt, für die eine Neuauflage bereits durchgeführter Förderprogramme keine Option darstellt, weshalb neue Themen und Begriffe Vorzug erhalten. Hier schließt sich ein Kreis: In der Latenzphase kann das Thema auf etablierte Diskurse aufsatteln, in der Fokusphase ist das Thema selbst der diskursive Horizont, innerhalb dessen verschiedene Fragestellungen prominent positioniert werden können, während schließlich in der Normalisierungsphase bisherige Teilaspekte eigene Karrieren entwickeln (siehe Abb. 9.5).

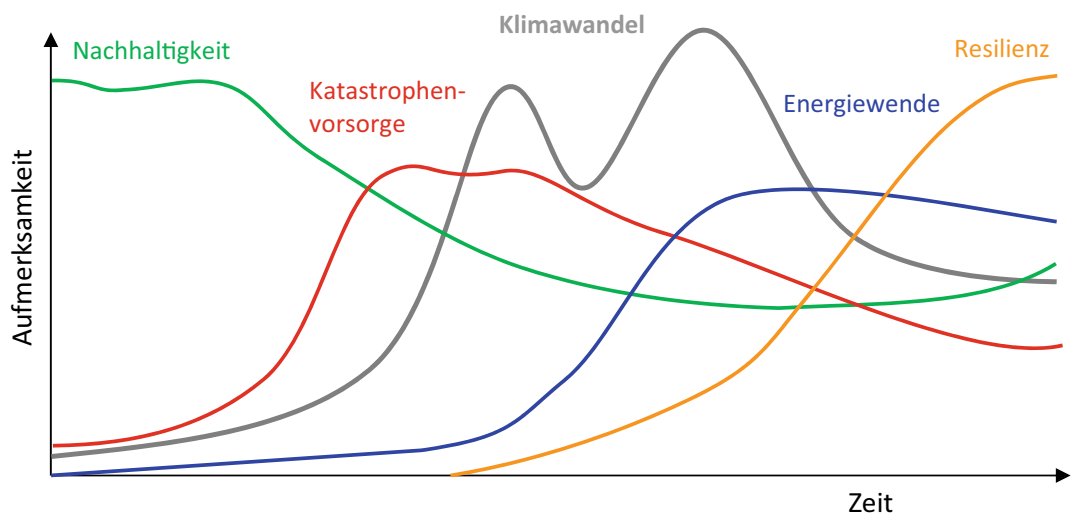

Abb.9.5 Mögliche Aufmerksamkeitskurven interagierender Themenkarrieren (Quelle: Eigene Darstellung (grobe Schätzungen markanter Subthemen des Klimawandeldiskurses ohne empirische Basis))

\section{Fazit}

Nach der Fokusphase erlebt die Verhandlung des Themas durch die Disziplin eine Normalisierung. Das heißt, die zwischenzeitliche Dynamik der Themenkarriere 
stabilisiert sich auf einem gegenüber der Latenzphase erhöhten Aufmerksamkeitsniveau. Bisherige Subthemen geraten nun in den Fokus, sodass der Diskurs in verschiedene Kommunikationszusammenhänge zerfällt. Allerdings hat sich das Thema in die fachgemeinschaftliche Wissens- und Sozialstruktur sowie in die individuellen Forschungsbiografien nachhaltig eingeschrieben. Innerhalb der Disziplin ist das Thema weiterhin in Lehrstühlen, Curricula und Resourcenallokationen manifestiert. Auch die Institutionen der Planungspraxis und -politik wurden im Hinblick auf das Thema langfristig transformiert. Das Verhältnis des Themas zur Fachöffentlichkeit hat sich gewandelt. Es wird nun leichter Aufmerksamkeit erwecken als Themen, die noch keine solche Karriere durchlaufen haben.

\subsection{Mechanismen der wissenschaftlichen Themenentstehung: Stabilität und Wandel}

In diesem Kapitel wird auf die zugrundeliegenden Mechanismen wissenschaftlicher Themenkarrieren eingegangen. Während die vorangegangene Beschreibung der Themenentstehung eine Synopse der empirischen Kapitel darstellt, werden im Folgenden verstärkt die wissenschaftssoziologischen, politik- und kommunikationswissenschaftlichen Theorien der Entstehung von Aufmerksamkeit (siehe Kp. 2 und 3) aufgegriffen, um zu prüfen, inwieweit durch sie die anfängliche Vernachlässigung von Themen, die plötzliche Fokussierung sowie die anschließende Normalisierung erklärt werden kann.

Themenkarrieren sind durch Aufmerksamkeitsverteilungen determiniert. Um die Entstehung von Themen erklären zu können, müssen also die Ursachen für das zunächst niedrige Niveau, den folgenden raschen Anstieg, die mehrjährige Fokussierung sowie das anschließende Absinken der Aufmerksamkeit gefunden werden. Aufmerksamkeit gegenüber einem Thema lässt sich anhand der formalisierten Kommunikation bestimmen, die sich im System Wissenschaft in Publikationen und Zitationen manifestiert. Ihr zugrunde liegt das Maß, in welchem Wissenschaftler*innen ihre begrenzten Forschungskapazitäten auf das Thema verwenden. Zur Erklärung von Themenkarrieren ist also möglichst die Veränderung der aggregierten Themenwahl der planungswissenschaftlichen Fachgemeinschaft im Rahmen ihrer Forschung zu rekonstruieren.

Für die wissenschaftliche Themenwahl sind die individuellen Handlungsressourcen und Handlungsorientierungen von Wissenschaftler*innen sowie der institutionelle Kontext maßgeblich, der sich aus der fachgemeinschaftlichen 
Wissensproduktion und den Steuerungsmechanismen (siehe Abb. 5.3) zusammensetzt. Grundsätzlich zeichnet sich die wissenschaftliche Themenwahl durch eine hohe Pfadabhängigkeit bzw. Stabilität aus: Wissenschaftler*innen müssen sich spezialisieren, um den kollektiven Wissensstand in einem bestimmten Bereich ergänzen zu können. Ein neues Thema wissenschaftlich aufzugreifen ist für sie mit großem Aufwand verbunden, weshalb vor allem Akteur*innen, die noch keine Spezialisierung entwickelt haben, hierfür in Frage kommen. Akteur*innen dagegen, die sich bereits in einem Feld etabliert haben, die sich also epistemisches, soziales, ökonomisches und politisches Kapital erarbeitet haben, werden eher davor zurückschrecken, ein gänzlich neues Feld zu erschließen. Würden die Mitglieder der Fachgemeinschaft allein auf Basis ihres Forschungsportfolios Themenentscheidungen treffen, wären abrupte Fokusverschiebungen innerhalb der Disziplin äußerst ungewöhnlich.

Die disziplinäre Vernachlässigung des Themas in der Latenzphase kann durch diese individuelle und fachgemeinschaftliche Stabilität des Forschungsportfolios partiell erklärt werden: Um ein Thema im Rahmen der eigenen Forschung aufgreifen zu können, muss es zunächst als solches wahrgenommen werden. Innerhalb etablierter Wissens- und Sozialstrukturen - auch „Paradigma“ (Kuhn) oder „multiparadigmatische Struktur" (Kornmesser und Schurz) -, in denen die Akteur*innen ihre jeweiligen Forschungsprogramme bearbeiten, ist die Aufmerksamkeit jedoch auf ein bereits bestehendes Problemset ausgerichtet. Selbst wenn wissenschaftliche Akteur*innen das Thema als raumplanungsrelevant wahrnehmen, so ist das systematische Aufgreifen im Rahmen der jeweils eigenen Forschungen noch nicht die logische Konsequenz. Angesichts aktueller Kooperationsanfragen, dringlicher Abgabefristen sowie langfristiger Forschungsprogramme ist es sogar unwahrscheinlich, dass Forschende dem neuen Thema Ressourcen widmen. Die meisten Planungswissenschaftler*innen erkennen keine Schnittmengen ihres Forschungsportfolios mit dem neuen Thema und vermeiden den Aufwand, es neu zu erschließen. Auch etablierte Akteur*innen, die das Thema als besonders relevant für ihre Spezialisierung anerkennen, reagieren zurückhaltend oder widerwillig. Indem es etablierte Wissensstrukturen in Frage stellt, sehen sie ihr wissenschaftliches Kapital - ihr Wissen, ihre Reputation, ihren Zugang zu finanziellen Mitteln - in Gefahr. Teilweise ergreifen sie deshalb sogar Maßnahmen zur Verhinderung des neuen Themas. Wenn das Thema dennoch wissenschaftlich bearbeitet wird, dann zumeist von Akteur*innen und Institutionen, die sich explizit auf das Aufspüren neuer raumrelevanter Entwicklungen spezialisiert haben - bspw. Qualifizierungsarbeiten, die laufende Raumbeobachtung oder Wissenschaftler*innen, die sich eher über ihre Rolle als Impulsgeber*innen 
denn als Spezialist*innen definieren. Allerdings erhalten ihre Vorstöße aus den genannten Gründen geringe Resonanz.

Warum kommt es dennoch zum Durchbruch des Themas? Die Durchbruchphase beschreibt den rasanten Wandel des institutionellen Kontextes von der strukturellen Vernachlässigung und Tabuisierung des Themas hin zur Priorisierung im Rahmen der fachgemeinschaftlichen Vermittlung von Wissensbedarfen sowie der Allokationen von finanziellen Ressourcen und Positionen.

Grundsätzlich ist es möglich, dass die Wissenschaft selbst diesen Wandel erzeugt. Kuhn schildert einen solchen Mechanismus: Im Zuge der ,normalen Wissenschaft" werden mehr und mehr Anomalien registriert, was schließlich in der Krise des alten und in der Durchsetzung eines neuen Paradigmas resultiert (siehe Kp. 2.1). Darauf aufbauend legen bspw. Crane und Mullins dar, wie eine neue Theorie, Idee oder ein neues Paradigma in der Disziplin durch persönlichen Kontakt diffundiert (siehe Kp. 2.3). Diese Prozesse sind allerdings mit einem längeren Zeitraum und mindestens einem Generationenwechsel verbunden. Die inkrementelle, nicht abreißende Diffusion des Themas Klimawandelanpassung im Zuge der Latenzphase kommt diesen Schilderungen am nächsten. Für die Erklärung des abrupten Durchbruchs des Themas im Jahr 2007 sowie des Verlaufs des Schrumpfungsthemas reichen diese wissenschaftssoziologischen Theorien jedoch nicht aus.

Stattdessen erinnern die Beobachtungen eher an Phänomene, die in den Agenda Setting-Forschungen beschrieben werden. So hat insbesondere das Punctuated Equilibrium Modell in Bezug auf die Stabilität etablierter Deutungen starke Ähnlichkeiten mit den wissenschaftssoziologischen Theorien, allerdings erfolgt der Politikwandel bzw. Paradigmenwechsel hier in Form plötzlicher Disruptionen. Fachbereiche lassen sich in diesem Sinne als stabile Wissens- und Sozialstrukturen (=> ,policy monopolies") beschreiben, innerhalb derer sich eine Konstellation aus Entscheidungsträger*innen weitgehend einig über das Framing bzw. die Deutung eines bestimmten wissenschaftlichen Gegenstandes ist (=>, policy image“). Indem diese Gemeinschaft über die Allokation von Reputation, finanziellen Ressourcen und Positionen entscheidet, kann sie ihre Wissensansprüche verteidigen und rivalisierende Deutungen zunächst marginalisieren. Irgendwann wird der Druck jedoch zu groß und die etablierte Deutung, die bislang von einem Großteil der Fachgemeinschaft für selbstverständlich gehalten wurde, wird zunehmend hinterfragt. Es zeichnet sich Gestaltungsspielraum in Bezug auf das Framing ab und bislang vernachlässigte Dimensionen des Themas rücken in den Fokus. Dies ruft zahlreiche bislang unbeteiligte Akteur*innen auf den Plan, die ihre alternativen Fragestellungen, Forschungsansätze und Deutungen einbringen und durchsetzen wollen. Es wird ein abrupter Paradigmenwechsel in Gang gesetzt, 
im Zuge dessen auch Ressourcenallokationen neu verhandelt und Institutionen modifiziert werden.

Im Rahmen der beiden untersuchten Themenkarrieren ist es jeweils eine hegemoniale Deutung, die im Zuge der Durchbruchphase disziplinweit ins Wanken gerät und schließlich aufgegeben werden muss. So wird der Glaube, Schrumpfung könne mit dem bewährten Instrumentarium bekämpft und schließlich in erneutes Wachstum umgekehrt werden, im Zuge der Durchbruchphase von der Erkenntnis abgelöst, Schrumpfung sei nicht abwendbar und müsse gesteuert, abgefedert und gestaltet werden. Das Thema Klimawandel erlebt einen solchen Paradigmenwechsel, als sich die Deutung durchsetzt, die Klimawandelanpassung sei ein unausweichliches Erfordernis, das nicht länger aus Sorge, Anstrengungen im Bereich Klimaschutz zu konterkarieren, aufgeschoben werden könne. In beiden Fällen geht der Paradigmenwechsel damit einher, dass eine an die neue Deutung geknüpfte Befürchtung sich - zumindest für einen Großteil der Fachgemeinschaft - in eine Versprechung verkehrt: Zwar erfordert der Paradigmenwechsel das Eingeständnis eines Scheiterns, dies impliziert aber nicht, dass das sich öffnende Betätigungsfeld für andere Akteur*innen und Instrumente geräumt werden muss. Im Gegenteil: Mehr und mehr wird deutlich, dass es gerade die Raumplanung ist, die einen Bedeutungsgewinn erwarten kann. Dass das Wissen und Instrumentarium der Planungswissenschaft im Zuge des Paradigmenwechsels einer Revision und Erweiterung bedarf, ist für die Disziplin überwiegend mit Vorteilen verbunden. So steigt das Interesse an planungswissenschaftlichen Erkenntnissen, es werden Ressourcen verfügbar und es kann Einfluss auf die Planungspraxis genommen werden.

An dieser Stelle bietet sich auch ein Rückgriff auf Kingdons policy windows an. Sie öffnen sich, wenn drei voneinander unabhängige Ströme - Lösungen, Entscheidungsprozesse und Problemwahrnehmung - sich kreuzen. So steigt die fachöffentliche Aufmerksamkeit gegenüber dem Thema Klimawandel erst dann rasant an, als mehr und mehr Akteur*innen im Jahr 2007 zu der Überzeugung gelangen, dass die Raumplanung ganz entscheidend zur Anpassung an den Klimawandel beitragen könne, als also eine wachsende Problemwahrnehmung mit dazu passenden Lösungsansätzen aus dem Feld der Planung verknüpft wird. Für das Thema Schrumpfung scheint dieser Rückgriff allerdings weniger ertragreich, da die Problemwahrnehmung zumindest in Teilen der Planungswissenschaft bereits seit den 1970er-Jahren mit kompatiblen Lösungsansätzen gekoppelt war. Erst als die Politik das Thema aufgrund unübersehbarer ökonomischer und politischer Auswirkungen aufgreift, vervielfacht auch die Planungswissenschaft schlagartig ihren wissenschaftlichen Output zu dem Thema. Auch der Durchbruch des Themas Klimawandel ist nicht der selbstreferenziellen Wissensproduktion der 
Disziplin zuzuschreiben. Die Auslöser der Fokussierung sind vielmehr außerhalb der planungswissenschaftlichen Disziplin zu suchen.

Es ist festzuhalten, dass die empirischen Befunde keine Anhaltspunkte dafür geben, dass allein wissenschaftliche Mechanismen eine kontinuierliche Akkumulation einschlägigen Wissens gewährleisten, geschweige denn eine disziplinäre Fokussierung auslösen können. Insbesondere für die zwischenzeitliche Intensivierung der einschlägigen Forschung im Rahmen der beiden erforschten Themenkarrieren sind stattdessen externe Impulse verantwortlich zu machen. Trotz in der Nische bereits vorliegender Erkenntnisse und möglicher Politikansätze zur Problemlösung herrschen bis zum Durchbruch geringes fachöffentliches Interesse, strukturelle Benachteiligung und Widerstreben gegenüber dem neuen Thema vor. Dieser Zustand und die ihm zugrundeliegenden Mechanismen werden erst beendet und schließlich in ihr Gegenteil transformiert, als die Massenmedien, die Politik und die Wirtschaft das Thema auf die Agenda setzen. Durch eine Häufung von Ereignissen - politische Prozesse, Auftragsforschungen, gerichtliche Entscheidungen uvm. - wird das Feld der Raumplanung in eine Irritation versetzt. Verschiedene Akteur*innen der Planungspraxis, -politik und forschung erkennen die potenzielle Bedeutung des Themas. Sie beginnen im Rahmen von Forschungsaufträgen, Kommissionen, Gutachten, Arbeitskreisen und Workshops den Handlungs- und Wissensbedarf für die nächsten Jahre zu eruieren und schließlich festzulegen. Vermittelt über verschiedene strukturelle Kopplungen werden Handlungserfordernisse im Feld der Raumplanung in wissenschaftliche Forschungslücken und daraus resultierende Forschungsprogramme übersetzt. Eine zentrale Rolle spielen hierbei weithin wahrnehmbare Förderprogramme der angewandten Wissenschaft: Innerhalb weniger Jahre treten verschiedene Geldgeber*innen auf den Plan, wobei mögliche inhaltliche Überschneidungen zwischen den Förderprogrammen kein Hemmnis, sondern eher ein Katalysator für deren Initiierung darstellen.

Das plötzlich einsetzende externe Interesse erweitert zum einen den bislang von theoretischen und präskriptiven Überlegungen geprägten Forschungsgegenstand maßgeblich um Fragen der politischen Regulierung, der planungspraktischen Umsetzung und der Erfolgskontrolle. Zum anderen setzt es wissenschaftsinterne Mechanismen und selbstverstärkende Effekte in Gang. Durch den systemexternen Handlungsbedarf und die aufgelegten Förderprogramme wird die bisherige Nische (bzw. werden verschiedene bisherige Nischen) für mehrere Jahre zu einem wachsenden Feld. Nicht nur die Verfügbarkeit finanzieller Ressourcen ist deshalb in diesem Feld vergleichsweise hoch, sondern auch die zunehmende Aufmerksamkeit seitens der Disziplin ist gesichert. Akteur*innen, die bereits zu dem Thema geforscht haben, können jetzt ihre Erkenntnisse verwerten und 
erhöhen ihre Sichtbarkeit durch eine steigende Publikationsfrequenz. Zahlreiche Wissenschaftler*innen und Forschungsgruppen treten zusätzlich in den Diskurs ein und machen mit Vorträgen, Publikationen und Fördermittelanträgen auf sich aufmerksam. Sie verknüpfen ihre Forschungsportfolios mit dem neuen Thema, was sowohl neue Erkenntnisse schafft als auch neuen Forschungsbedarf offenlegt. Zwar entsteht nun ein Resonanzboden, der es ermöglicht, Reputation und Ressourcen zu erwerben, es entwickelt sich aber auch eine Konkurrenzsituation im Wettbewerb um Aufmerksamkeit und zur Verfügung gestellte Ressourcen, die zu einem erhöhten wissenschaftlichen Output animiert. Der zuvor in der Nische geringe Zeitdruck in Bezug auf die Veröffentlichung wissenschaftlicher Ergebnisse nimmt schlagartig zu.

Der externe Einfluss auf die Planungswissenschaft zeigt sich nicht zuletzt in der Doppelpeakstruktur der Aufmerksamkeitskurven (siehe Abb. 9.1 und Abb. 9.3). Während des ersten Höhepunktes dominiert inhaltlich die theoretische Erschließung und Übersetzung des Themas, im zweiten Höhepunkt tritt hingegen die Veröffentlichung von Ergebnissen aus Forschungsprojekten und empirischen Fallstudien in den Vordergrund. Nach dem zweiten Höhepunkt sinkt die disziplinäre Aufmerksamkeit ab, was wiederum zeitlich mit dem Rückgang des systemexternen Interesses korreliert bzw. genauer: Der zweite planungswissenschaftliche Aufmerksamkeitshöhepunkt erfolgt, nachdem bereits eine Ermüdung der gesellschaftlichen Aufmerksamkeit, eine Reduktion des externen Wissensbedarfs und das Auslaufen der großen Förderprogramme zu verzeichnen sind. Der Grund für die Verzögerung ist einerseits in der längeren Reaktionszeit der Wissenschaft zu sehen, andererseits in den unterschiedlichen Handlungslogiken der Systeme: Während Medien und Politik an einem schnellen Abschluss des Problems - unabhängig von dessen tatsächlicher Lösung - interessiert sind (,,looking for closure"), verfolgt die Wissenschaft auf methodisch kontrollierte Weise die Gewinnung langfristig gültiger Erkenntnisse. Während Medien und Politik die Komplexität der Realität systematisch reduzieren, um Verständlichkeit zu gewährleisten und zu Entscheidungen gelangen zu können, bietet die Wissenschaft den Raum, um in hochspezialisierten Expert*innenkreisen die unterschiedlichen Facetten des Problems zu erforschen.

Dass insbesondere die Planungswissenschaft aber eben nicht unabhängig von unmittelbaren gesellschaftlichen Nutzenerwartungen und Relevanzkriterien operiert, zeigt nicht zuletzt die Normalisierungsphase. Der abnehmende externe Wissensbedarf und die Reduktion der Drittmittel für einschlägige Forschung lösen wiederum selbstverstärkende Effekte in der Disziplin aus. Die Wachstumsspirale der Durchbruch- und beginnenden Fokusphase wird umgedreht: Jetzt konkurriert eine große Zahl einschlägiger Expert*innen um eine sinkende Nachfrage 
außerhalb und innerhalb der Planungswissenschaft. Nicht nur der Erwerb von Drittmitteln und Positionen ist in dem Themenfeld jetzt vergleichsweise schwierig, auch hat der verbliebene und neu hinzukommende Forschungsbedarf nicht mehr die hohe Bedeutung, die er zu Beginn der Fokusphase hatte. Die Konkurrenz um Drittmittel und potenzielle Wissensansprüche in dem Feld ist deshalb im Vergleich zu anderen Themen höher, weshalb die Forscher*innen neue Verwertungsmöglichkeiten für ihr Wissen suchen und sich anderen Themen zuwenden. Auch geraten jetzt Detailfragen und vormalige Subthemen in den Blick, weshalb das zuvor fokussierte überspannende Thema durch neue Begriffe und Frames ersetzt wird.

\subsection{Auswirkungen von Themenkarrieren auf die Wissenschaft: Gekommen, um zu bleiben}

Temporäre Fokussierungen von Themen verändern die disziplinäre Wissensproduktion in vielen Bereichen tiefgreifend und nachhaltig. Inhaltliche und soziale Gesichtspunkte gehen dabei Hand in Hand.

Die im Rahmen dieser Arbeit untersuchten Themenkarrieren wurden maßgeblich durch politische und planungspraktische Relevanzkriterien und Nutzenerwartungen bestimmt. Zunächst ist daher festzuhalten, dass die Wissenschaft den systemexternen Bedarf, teilweise mit einer Verzögerung von wenigen Jahren, weitgehend befriedigen konnte. Die Raumplanung und die Planungswissenschaft konnten dadurch nicht nur ihre gesellschaftliche Relevanz unter Beweis stellen, sondern auch einen Bedeutungsgewinn verzeichnen. Einschlägige planungswissenschaftliche Expertise hat sich seit dem Durchbruch der Themen nicht nur im Rahmen von Strategiepapieren, Leitbildern, Förderprogrammen, Plänen, Gesetzen sowie organisationalen und personellen Anpassungen institutionalisiert. Auch in der gebauten Umwelt findet die vorübergehende Fokussierung ihren dauerhaften Niederschlag. Insofern lassen sich temporäre Fokussierungen von Themen im Feld der Raumplanung als strukturelle Kopplungen zwischen Wissenschaft und Praxis verstehen: Die Wissenschaft erhält Finanzmittel und die Möglichkeit, Einfluss auf Planung und Gesetzgebung zu nehmen. Im Gegenzug produziert und kommuniziert sie spezifisches Wissen ${ }^{1}$.

Die schnelle Befriedigung des externen Legitimations- und Wissensbedarfs im Zuge der Durchbruch- und Fokusphase bringt tiefgreifende Anpassungen in der

\footnotetext{
${ }^{1}$ Auch Luhmann weist in „Die Realität der Massenmedien“ (1996: 28) auf die Funktion von Themen hin, verschiedene Bereiche der Gesellschaft strukturell zu koppeln.
} 
disziplinären Wissensproduktion und Sozialstruktur mit sich. Bestimmte Kommunikationskanäle bzw. Schnittstellen zwischen Wissenschaft und Systemumwelt, die sich als besonders effektiv erweisen, gewinnen an Einfluss. Die an ihnen beteiligten Planungswissenschaftler*innen bündeln, sortieren und hierarchisieren den rasch wachsenden Wissensbestand für die Bedarfe der Systemumwelt und wirken bei der Festlegung weiterer Wissensbedarfe mit. Bei der Gestaltung und Umsetzung von Förderprogrammen, Strategiepapieren oder Gesetzesänderungen erhalten sie eine herausgehobene Bedeutung. Diese vorübergehende Vermittlungsoder auch Gatekeeping-Funktion verschafft ihnen Informations- und Kontrollvorteile, sichert ihnen ökonomisches, politisches und soziales Kapital und damit einen langfristigen Bedeutungsgewinn in der Wissenschaft.

Auch abseits dieser Gatekeeper*innen zwischen Wissenschaft und Systemumwelt manifestiert sich die vorübergehende Fokussierung nachhaltig in der Allokation von Reputation und Einfluss. Im Rahmen von Drittmittelvergaben, Arbeitskreisen, Themenheften, Sammelbänden und Konferenzen wird insbesondere denjenigen Akteur*innen Raum gegeben, die an das Thema anknüpfen können, was eine gleichzeitige „Diskriminierung“ (Luhmann 1991: 213) jener Akteur*innen bedeutet, die keinen Beitrag leisten können. Auf individueller Ebene erhalten vorübergehend einschlägige Fragestellungen mehr Beachtung, während Forschungspfade, die sich nicht mit dem fokussierten Thema verknüpfen lassen, vernachlässigt werden. Auch bei der Besetzung von Stellen und leitenden Positionen in der Planungswissenschaft und der Planungspraxis haben einschlägig forschende Akteur*innen einen Vorteil: Sie verfügen durch die Fokussierung zum einen über eine erhöhte wissenschaftliche Autorität. Zum anderen passen die Einrichtungen ihre inhaltliche Ausrichtung im Zuge der Fokussierung an, was sich in veränderten Auswahlkriterien für Auftragsvergaben, Stellenbesetzungen und Beförderungen manifestiert. Während sich diese Auswahlkriterien nach der Fokussierung verflüchtigen, ist die Besetzung einflussreicher Positionen und die von ihnen ausgehende Bevorzugung bestimmter Themen - bspw. in der Lehre, in Begutachtungen und in weiteren Stellenbesetzungen - von langfristiger Natur.

Themenkarrieren modifizieren die disziplinäre Netzwerkstruktur: Die einschlägigen Konferenzen, Arbeitskreise und Forschungsprojekte beeinflussen, wer sich kennenlernt, welche Form der Austauschbeziehung miteinander eingegangen wird und welches Sozialkapital aus den Beziehungen resultiert. In einem bestimmten Themenbereich erweitert und verdichtet sich das persönliche Netzwerk kooperierender Forscher*innen im Zuge der disziplinären Fokussierung besonders stark, während andere Themenbereiche vorübergehend weniger Kooperationsaktivität aufweisen. Indem thematische Nischen in das Zentrum der disziplinären Aufmerksamkeit gehoben werden, gewinnen deren Mitglieder an 
Status: Nicht nur nehmen zentrale Akteur*innen der Disziplin Beziehungen mit ihnen auf, auch gewinnen bereits bestehende Kooperationspartner*innen an Zentralität. Um also akute Wissensbedarfe innerhalb und außerhalb der Disziplin schnell und umfassend bedienen zu können, wird die disziplinäre Sozialstruktur nachhaltig transformiert.

Disziplinäre Fokussierungen sorgen in einer zunehmend ausdifferenzierten Wissenschaft für den wechselseitigen Austausch zwischen und die temporäre Integration von Fachgemeinschaften und Wissensarten. Akteur*innen unterschiedlicher disziplinärer Fachbereiche prüfen im Zuge des Durchbruchs und der Fokussierung, ob ihr Forschungsportfolio Wissensbezüge und Kooperationen bereithält, die sich auf das neue Thema anwenden lassen. Dadurch werden Silos isolierter Forschung aufgebrochen und das Spezialwissen wird für die anderen - durch die gemeinsame Kommunikation über das fokussierte Thema vorübergehend besonders interessierten - Fachbereiche zugänglich gemacht. Dies gilt insbesondere für jene Nischen, die in das Zentrum der disziplinären Aufmerksamkeit gehoben werden. Ihr über Jahre angehäuftes Wissen kommt (erst) jetzt der gesamten Disziplin zugute und ihre hochspezialisierten Beziehungen werden um Kontakte mit für sie neuen thematischen Facetten ergänzt, was wiederum neue Erkenntnisse befördert. Die Disziplin wird also durch temporäre Fokussierungen in Bewegung gehalten, durchmischt und vernetzt. Der integrierende Effekt von Themenkarrieren gilt nicht allein disziplinintern: Planungspraktische Erfordernisse, die womöglich in der Nische schon lange bekannt sind, sind nun für alle erkennbar, was den Expert*innen Gehör in der Systemumwelt verschafft und dem Spezialwissen zu planungspraktischer Umsetzung verhilft.

Fokussierungen induzieren die rasche Erweiterung des Wissens in bestimmten Bereichen, bei gleichzeitiger Vernachlässigung anderer Bereiche. Bei Einsetzen der Normalisierungsphase nimmt der Wissensbedarf der Systemumwelt ab und auch innerhalb der Wissenschaft gelten die wichtigsten Fragen vorübergehend als beantwortet. Dennoch hat die punktuelle Erweiterung eine nachhaltig veränderte disziplinäre Schwerpunktsetzung zur Folge. Die bereits benannten, hierfür maßgeblichen sozialen Mechanismen haben auch eine epistemische Komponente: Innerhalb der Wissenschaft erzeugt die Anhäufung von Wissen keine Sättigung, sondern lässt stattdessen das Nichtwissen anwachsen oder zumindest sichtbar werden (Weingart 2003: 9-10). Mehr Forschung in einem bestimmten Feld zieht also nicht unbedingt eine Reduktion, sondern eher eine Vermehrung der Fragen in eben diesem Feld nach sich. Dieser epistemische Mechanismus lässt sich mit den medien- und kommunikationswissenschaftlichen Agenda SettingAnsätzen ergänzen: Die im Zuge der Fokussierung erhöhte Salienz eines Themas 
beeinflusst nachhaltig, welche Inhalte für wichtig erachtet werden. Indem Wissenschaftler*innen über das Thema lesen, nachdenken, forschen und kommunizieren, messen sie ihm mehr Gewicht bei, was sich auf die zukünftige Wahrnehmung, Selektion und Beurteilung anderer Themen und Ereignisse auswirkt. Bestimmte Attribute neuer und altbekannter Themen werden nun mehr Aufmerksamkeit - in Publikationen, Begutachtungen, Lehrveranstaltungen, Diskussionen, Evaluationen, Entwürfen usw. - erhalten als andere. Letztlich werden dadurch nicht nur Relevanzkritieren verschoben, sondern auch persönliche Prioritäten, Bewertungsmaßstäbe und Einstellungen verändert, wodurch wiederum soziales Handeln und Entscheidungsprozesse strukturiert werden.

All diese Prozesse schreiben sich nachhaltig in die disziplinäre Wissens- und Sozialstruktur ein. Im Feld der Planungspraxis wird das Thema durch organisationale und personelle Anpassungen, Gesetzesänderungen, Förderprogramme und Pläne institutionalisiert. Auch räumliche und gesellschaftliche Strukturen sowie deren Perzeption durch die Raumplanung werden im Zuge der vorübergehenden Fokussierung verändert. Dieser Wandel des Forschungsgegenstandes wirkt sich wiederum in der Planungswissenschaft auf Ressourcenallokationen, Forschungsprogramme sowie die Ausbildung zukünftiger Raumplaner*innen aus. Zahlreiche Mitglieder der Disziplin akkumulieren im Zuge der Fokussierung epistemisches, soziales, ökonomisches und politisches Kapital. Indem diese Handlungsressourcen zu einem großen Teil thematisch gebunden sind, indem also beispielsweise die erworbene Reputation sich nur in spezifischen Fachgemeinschaften realisieren lässt, verändern sie die Selektionskriterien für zukünftige Forschungsprobleme. Die soziale Netzwerkstruktur der Disziplin wird transformiert, indem ausgewählte Bereiche, jetzt um zahlreiche Akteur*innen und wichtige Beziehungen erweitert, an Zentralität gewinnen. Dies gilt auch für das kognitive Netzwerk: Die Wissensstruktur der Disziplin wird jetzt um viele Erkenntnisse und Bezüge in einem bestimmten Feld erneuert und ergänzt. Nicht zuletzt die Wahrnehmungen, Bewertungsmaßstäbe und persönlichen Einstellungen der gesamten Disziplin verschieben sich deshalb zugunsten des vorübergehend fokussierten Themenfeldes.

Auch wenn das Thema in der Normalisierungsphase nicht mehr im Fokus der Disziplin steht, sein Verhältnis zur fachöffentlichen Aufmerksamkeit ist nun ein anderes. Es wird leichter Interesse erwecken als Themen, die noch keine Karriere durchlaufen haben. Das Thema wird zum Kontext, vor dessen Hintergrund neue Probleme wahrgenommen, gerahmt und gelöst werden. Es gilt der Satz von Gläser und Laudel (2015a: 302) „Previous research influences the choice of new research problems, as current research will - through modifying the structures - influence future choices", allerdings nicht allein in Bezug auf 
Wissensstrukturen und nicht nur auf Ebene individueller Forschungsbiografien: Indem Themenkarrieren die Wissens- und Sozialstruktur der gesamten Disziplin modifizieren, beeinflussen sie, ob und in welcher Form sich ihre Mitglieder in Zukunft bestimmten Themen zuwenden.

Open Access Dieses Kapitel wird unter der Creative Commons Namensnennung 4.0 International Lizenz (http://creativecommons.org/licenses/by/4.0/deed.de) veröffentlicht, welche die Nutzung, Vervielfältigung, Bearbeitung, Verbreitung und Wiedergabe in jeglichem Medium und Format erlaubt, sofern Sie den/die ursprünglichen Autor(en) und die Quelle ordnungsgemäß nennen, einen Link zur Creative Commons Lizenz beifügen und angeben, ob Änderungen vorgenommen wurden.

Die in diesem Kapitel enthaltenen Bilder und sonstiges Drittmaterial unterliegen ebenfalls der genannten Creative Commons Lizenz, sofern sich aus der Abbildungslegende nichts anderes ergibt. Sofern das betreffende Material nicht unter der genannten Creative Commons Lizenz steht und die betreffende Handlung nicht nach gesetzlichen Vorschriften erlaubt ist, ist für die oben aufgeführten Weiterverwendungen des Materials die Einwilligung des jeweiligen Rechteinhabers einzuholen.

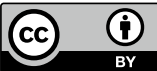

